\title{
Role of defective calcium regulation in cardiorespiratory dysfunction in Huntington's disease
}

\author{
Haikel Dridi, ${ }^{1}$ Xiaoping Liu, ${ }^{1}$ Qi Yuan, ${ }^{1}$ Steve Reiken, ${ }^{1}$ Mohamad Yehya, ${ }^{2}$ Leah Sittenfeld, ${ }^{1}$ \\ Panagiota Apostolou, ${ }^{1}$ Julie Buron, ${ }^{3}$ Pierre Sicard, ${ }^{2}$ Stefan Matecki, ${ }^{2}$ Jérome Thireau, ${ }^{2,4}$ \\ Clement Menuet, ${ }^{3}$ Alain Lacampagne, ${ }^{2,4}$ and Andrew R. Marks ${ }^{1}$ \\ 'Department of Physiology and Cellular Biophysics, Clyde and Helen Wu Center for Molecular Cardiology, Columbia \\ University Vagelos College of Physicians and Surgeons, New York, New York, USA. ${ }^{2}$ PHYMEDEXP, University of Montpellier, \\ CNRS, INSERM, CHRU Montpellier, Montpellier, France. Institut de Neurobiologie de la Méditerranée, INMED UMR1249, \\ INSERM, Aix-Marseille Université, Marseille, France. ${ }^{4} \mathrm{LIA}$ MusCaRyR, CNRS, Montpellier, France.
}

\begin{abstract}
Huntington's disease (HD) is a progressive, autosomal dominant neurodegenerative disorder affecting striatal neurons beginning in young adults with loss of muscle coordination and cognitive decline. Less appreciated is the fact that patients with HD also exhibit cardiac and respiratory dysfunction, including pulmonary insufficiency and cardiac arrhythmias. The underlying mechanism for these symptoms is poorly understood. In the present study we provide insight into the cause of cardiorespiratory dysfunction in HD and identify a potentially novel therapeutic target. We now show that intracellular calcium $\left(\mathrm{Ca}^{2+}\right)$ leak via posttranslationally modified ryanodine receptor/intracellular calcium release (RyR) channels plays an important role in HD pathology. RyR channels were oxidized, PKA phosphorylated, and leaky in brain, heart, and diaphragm both in patients with HD and in a murine model of HD (Q175). HD mice (Q175) with endoplasmic reticulum $\mathrm{Ca}^{2+}$ leak exhibited cognitive dysfunction, decreased parasympathetic tone associated with cardiac arrhythmias, and reduced diaphragmatic contractile function resulting in impaired respiratory function. Defects in cognitive, motor, and respiratory functions were ameliorated by treatment with a novel Rycal small-molecule drug (S107) that fixes leaky RyR. Thus, leaky RyRs likely play a role in neuronal, cardiac, and diaphragmatic pathophysiology in HD, and RyRs are a potential novel therapeutic target.
\end{abstract}

Conflict of interest: Columbia University and ARM own stock in ARMGO Pharma, Inc., a company developing compounds targeting the ryanodine receptor, and have patents on Rycals (e.g., US 2014/0187536)

Copyright: (c) 2020, Dridi et al. This is an open access article published under the terms of the Creative Commons Attribution 4.0 International License.

Submitted: May 26, 2020

Accepted: September 2, 2020

Published: September 8, 2020.

Reference information: JCI Insight.

2020;5(19):e140614.

https://doi.org/10.1172/jci.

insight.140614.

\section{Introduction}

Huntington's disease (HD) is a progressive, autosomal dominant neurodegenerative disorder affecting striatal neurons in young adults with distinct symptoms of cognitive decline and muscle incoordination $(1,2)$. This devastating disease is usually fatal approximately 10-15 years after onset of symptoms, and there is no disease-modifying treatment. HD is caused by a mutation in the HTT gene on chromosome 4 encoding the huntingtin protein. CAG repeat expansion results in a polyglutamine region (poly Q) at its $\mathrm{N}$-terminus (3). Patients with HD exhibit impaired locomotor and respiratory muscle function and digestive tract dysfunction (4).

The leading cause of death in patients with $\mathrm{HD}$ is aspiration pneumonia $(5,6)$, due to dysphagia $(7,8)$ Indeed, diaphragmatic muscle weakness combined with difficulty clearing airway secretions and defective swallowing are prevalent in patients with $\operatorname{HD}(9,10)$. However, the mechanisms underlying the respiratory dysfunction remain unknown (11-13). Most patients with HD do not report respiratory symptoms until later stages of the disease, when impaired swallowing and respiratory muscle weakness cause fatal aspiration pneumonias $(7,11,14)$. At the late stages of $\mathrm{HD}, 44 \%$ of patients receive respiratory therapy compared with $2 \%$ at the early stages of the disease (12). In a recent study, patients with HD exhibited reduced respiratory pressure, forced vital capacity, peak expiratory flow, and maximal voluntary ventilation (13).

Heart failure occurs in approximately $30 \%$ of patients with HD (15), in contrast to less than approximately $2 \%$ of age-matched individuals in the general population (16-22). Despite the limited number of studies evaluating heart function in HD, epidemiological data identify cardiac disease as the second most common cause of death in HD. Recently Stephen et al. reported significant cardiac contractile dysfunction 
and ECG abnormalities in a large cohort of early symptomatic patients with $\mathrm{HD} ; 25.3 \%$ of them exhibit ECGs abnormalities including bradycardia and prolonged Qtc interval (23).

Autonomic nervous system (ANS) dysfunction is a feature of HD and may play a role in the increased risk of cardiac arrhythmias in $\mathrm{HD}(4,24,25)$. Patients with $\mathrm{HD}$ may exhibit reduced heart rate variability (HRV) and altered sympathetic and parasympathetic activity $(26,27)$. Decreased cardiovagal regulation was found in patients with HD characterized by reduced HRV at rest and during deep respiration (24). It has been suggested that dysautonomia results in fatal cardiac arrhythmias $(25,28)$, due to impaired cardiac parasympathetic and sympathetic signaling (29). The cardiorespiratory consequences and possible mechanisms of ANS dysfunction in patients with HD are poorly understood. Ironically, patients with HD with cardiorespiratory manifestations are often excluded from clinical studies.

Neurodegeneration in HD may be associated with impaired synaptic transmission $(29,30)$, reduced brain-derived neurotrophic factor $(31,32)$, mitochondrial dysfunction $(33,34)$, and altered calcium $\left(\mathrm{Ca}^{2+}\right)$ regulation (35-37). Increased intracellular $\mathrm{Ca}^{2+}$ concentration due to NMDA receptor activity or other $\mathrm{Ca}^{2+}$ sources including the inositol 1,4,5-trisphosphate receptor type 1 (IP3R1) and ryanodine receptor (RyR) may play a role in striatal neurodegeneration in HD (38-42).

RyRs are ubiquitous intracellular $\mathrm{Ca}^{2+}$ release channels expressed early in development (43) and required for the function of many organs including the heart, skeletal muscle, and synaptic transmission in the brain (44). Three RyR mammalian isoforms are known: RyR1 (45, 46), RyR2 (47), and RyR3 (48), which are classified as skeletal muscle, heart, and brain types, respectively (44), although all 3 forms are expressed in the brain. In skeletal muscle RyR activation is linked to voltage-gated calcium channels (49), and in other organs including the heart, RyRs are calcium-activated calcium release channels (44). RyRs are homotetrameric macromolecular protein complexes (50) that include 4 RyR protomers (565 $\mathrm{kDa}$ each), kinases (PKA, Ca2 ${ }^{+} /$ calmodulin kinase [CaMKII]), phosphatases (PP1, PP2A), calmodulin, and a phosphodiesterase (PDE4D3) (47). The RyR channel-stabilizing subunit calstabin (FKBP12) is critical for stabilizing the closed state of the channel and preventing a pathological leak of calcium $(45,51)$. Maladaptive cAMP-dependent kinase A-mediated (PKA-mediated) phosphorylation and redox-dependent modifications (cysteine nitrosylation and oxidation) (52-54) of RyRs have been linked to a loss of calstabin from the channel macromolecular complex (47, 55-57). RyR remodeling, in turn, results in impaired $\mathrm{Ca}^{2+}$ handling because of a pathological $\mathrm{Ca}^{2+}$ leak from the sarcoplasmic reticulum/ER (SR/ER) and is associated with multiple organ dysfunction, including cognitive impairment $(54,58)$, heart failure (59), and respiratory/locomotor muscle weakness $(47$, $58,60-65)$.

Defective RyR function has been reported in $\mathrm{HD}$, leading to elevated intracellular $\mathrm{Ca}^{2+}$ levels and reduced endoplasmic reticular $\mathrm{Ca}^{2+}$ stores in R6/2 striatal and cortical neurons (40). Moreover, RyR inhibitors have been shown to be neuroprotective in vitro and improve motor behavior in vivo in YAC128 mice $(37,40,66)$.

Because defective $\mathrm{Ca}^{2+}$ regulation has been well documented in $\mathrm{HD}$, but not understood mechanistically, we hypothesized that leaky neuronal RyR plays a role, especially in the cardiorespiratory pathology. We focused on the type 2 isoform of RyR (RyR2) because it is predominantly expressed in the brain (67). Indeed, we previously showed that oxidation, nitrosylation, and PKA phosphorylation of RyR2 results in leaky channels that contribute to the pathophysiology of posttraumatic stress disorder (54) and Alzheimer's disease (AD) $(58,60)$. The role of RyR2 remodeling in brainstem nuclei remains unknown but might be involved in cardiac and respiratory dysfunction, such as arrhythmias and diaphragm muscle weakness in HD.

$\mathrm{HD}$ is characterized by cognitive dysfunction and involuntary motor movements $(68,69)$. We sought to determine whether leaky neuronal RyR2 channels play a role in cognitive dysfunction associated with HD. In addition, using a novel Rycal drug (S107), which crosses the blood-brain barrier and fixes the leak in RyR2 channels $(54,58,70)$, we further determined whether inhibiting RyR2-mediated ER Ca ${ }^{2+}$ leak can improve cognitive function in a murine model of HD. S107 is a small molecule that stabilizes the RyR2-calstabin2 interaction and decreases RyR2 ER Ca ${ }^{2+}$ leak without affecting PKA phosphorylation, oxidation, and cysteine nitrosylation of the channel $(54,58,60)$.

\section{Results}

Neuronal RyR2 channels are leaky in patients with $H D$ and in a murine model of $H D$. To evaluate the remodeling and functional abnormalities of RyR 2 in the brains of HD patients, cortical and hippocampal biopsies from deidentified organ donors (Supplemental Table 1; supplemental material available online with this article; https://doi.org/10.1172/jci.insight.140614DS1) with neuropathological grade 3 and 4 HD, 5 female and 3 
male (ages 54-66, CAG repeats 36-51), were obtained from the New York Brain Bank at Columbia University. We compared these specimens with cortical and hippocampal specimens from non-HD controls. ER fractions were purified to analyze the composition of the RyR2 macromolecular complex and posttranslational modifications known to be associated with channel leak (71) in the cortex and hippocampus (Figure 1, A-C). HD RyR2 exhibited PKA hyperphosphorylation (on RyR2-Ser2808), oxidation, and cysteine nitrosylation and were depleted of calstabin2 compared with controls. This is the "biochemical signature" of leaky RyR2 channels $(47,72)$. Single-channel recordings of cortical RyR2, reconstituted into planar lipid bilayers, revealed an increased open probability $\left(\mathrm{P}_{\mathrm{O}}\right)$ in the presence of low nonactivating $\left[\mathrm{Ca}^{2+}\right]_{\text {cis }}$ conditions under which normal RyR2 channels are tightly closed (Figure 1, D-G). This elevated $\mathrm{P}_{\mathrm{O}}$ is consistent with pathological $\mathrm{ER} \mathrm{Ca}^{2+}$ leak $(54,58)$. Indeed, neuronal microsomes from $\mathrm{HD}$ cortex and hippocampus exhibited increased RyR-mediated ER Ca ${ }^{2+}$ leak compared with controls (Supplemental Figure 1, A-D).

We evaluated the time course (at 2, 6, and 10 months of age) of RyR2 remodeling in 3 regions of the brain (striatum, cortex, and hippocampus) using 2 HD murine models (Q111 and Q175) compared with controls. We observed RyR2 remodeling in a time-dependent manner with optimal onset of oxidation, phosphorylation, and calstabin2 depletion being at 10 months of age (Supplemental Figure 2).

Then we chose the Q175 mouse model at 10 months old to evaluate RyR2 remodeling with and without S107 treatment compared with WT controls (littermates). As in HD patients' samples, RyR2 exhibited the biochemical signature of leaky channels in the cortex and in the hippocampus of Q175 mice compared with WT. RyR2 was PKA phosphorylated on Ser2808, oxidized, cysteine nitrosylated, and depleted of the stabilizing subunit calstabin2 (Figure 2, A-C). This RyR2 remodeling was associated with evidence of leaky RyR2 channels based on increased $\mathrm{P}_{\mathrm{O}}$ recorded at low nonactivating $\left[\mathrm{Ca}^{2+}\right]_{\text {cis }}$ in both the cortex and hippocampus of Q175 mice (Figure 2, D-K). Indeed, isolated brain mitochondria from Q175 mice exhibited increased reactive oxygen species (ROS) production, which may explain in part the oxidation of RyR2 channels (Supplemental Figure 3A). S107 administered in the drinking water reduced calstabin2 dissociation from the RyR2 macromolecular complex and decreased $\mathrm{ER} \mathrm{Ca}^{2+}$ leak (Figure 2, A-K).

Leaky RyR2 causes cognitive impairment in a murine model of $H D$. We assessed the effects of leaky RyR2 channels on spatial learning and memory using the Morris water maze (MWM) as previously described $(54,58)$. Q175 mice exhibited increased escape latency in the MWM at days 3 and 5 (Figure 3, A and B), spent less time in the target quadrant, and had a slightly reduced number of target crossings compared with WT mice (Figure 3, C and D). These findings are consistent with deficits in learning and/or memory. S107 treatment of Q175 mice significantly improved the escape latency and the time spent in the target quadrant compared with untreated Q175 mice, suggesting that RyR2 channel leak likely contributes to the cognitive deficits observed in the Q175 mice. Leaky RyR2 channels were also associated with increased anxiety determined using the elevated plus maze (EPM) test. Q175 mice spent more time and made more entries into the open arms of the EPM (Figure 3E). In the tail suspension test, Q175 mice exhibited increased immobilization duration consistent with increased stress, which was normalized by treatment with S107 (Figure 3F). The Q175 mice exhibited significantly impaired coordination in movement, suggesting deficits of motor neuron function. S107 treatment significantly improved the dysregulated movement coordination. Kyphosis testing and gait analysis did not reach significance, suggesting that the impaired motor neuron function was moderate (Supplemental Table 2). To confirm our results, we evaluated the RyR2 remodeling and the subsequent cognitive dysfunction in a second murine model of $\mathrm{HD}$ (R/6 mice) and observed similar results (Supplemental Figure 4 and Supplemental Table 3).

$R y R 2$ phosphorylation in autonomic brainstem areas affecting the heart. Although described as a disease of the central nervous system, studies have revealed abnormalities in non-neuronal organs in patients with HD (4). Whether these defects are a direct consequence of mutant huntingtin protein, or secondary to neurological dysfunction, is poorly understood. Cardiac function is regulated centrally via both parasympathetic and sympathetic activities. Cardiac vagal preganglionic neurons are located in the dorsal motor nucleus of the vagus (DMNV) and in the nucleus ambiguus (NA) in the medulla oblongata. A major source of cardiac sympathetic drive comes from adrenergic presympathetic $\mathrm{C} 1$ neurons located in the rostral ventrolateral medulla (73). C1 neurons are hyperactivated in multiple cardiovascular diseases, including heart failure, which might contribute to their etiology.

We evaluated the levels of RyR2 phosphorylation in NA and C1 neurons by immunohistochemistry in HD mice. Choline acetyltransferase and tyrosine hydroxylase staining were used as markers of NA and C1 neurons, respectively. Compared with WT, there was increased RyR2 phosphorylation at Ser2808 in the NA 


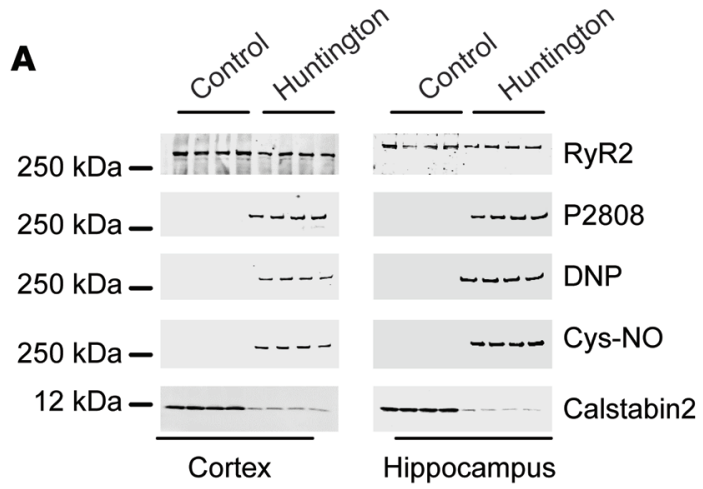

D Control

Po 0.01 To 0.96 ms Tc 144.4 ms
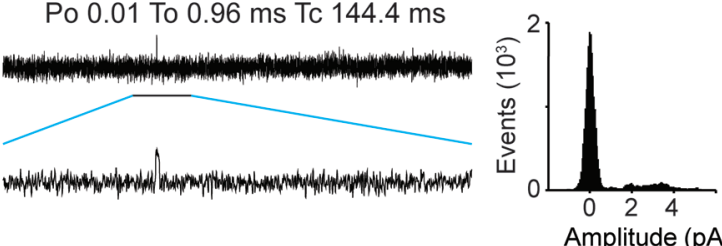

Patient 2

Po 0.11 To $4.98 \mathrm{~ms}$ Tc $5.0 \mathrm{~ms}$

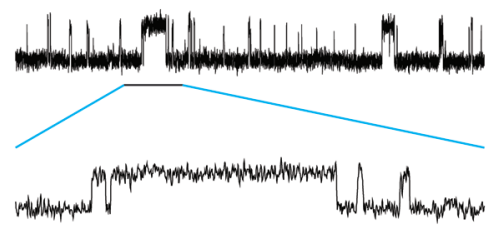

는

$1000 / 100 \mathrm{~ms}$
E

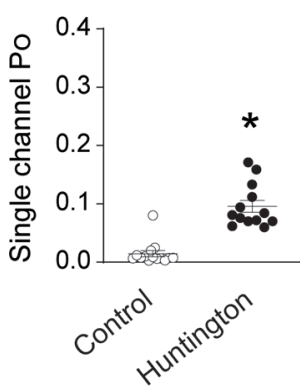

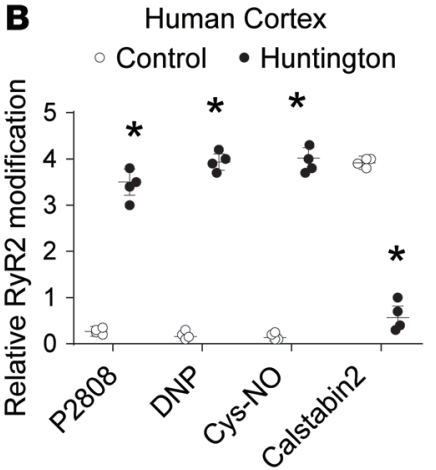

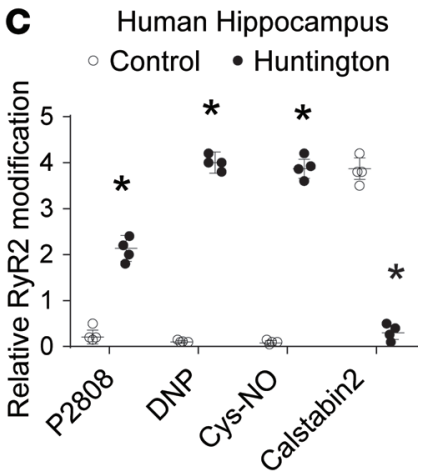

$[\mathrm{Ca}]_{\text {cyt }} 150 \mathrm{nM}$
Patient 1

Po 0.07 To $2.96 \mathrm{~ms}$ Tc $38.6 \mathrm{~ms}$
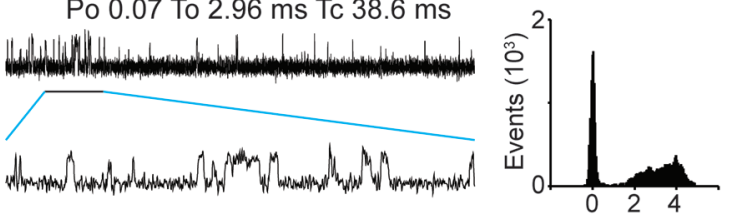

Patient 3

Po 0.08 To $3.57 \mathrm{~ms}$ Tc $23.6 \mathrm{~ms}$
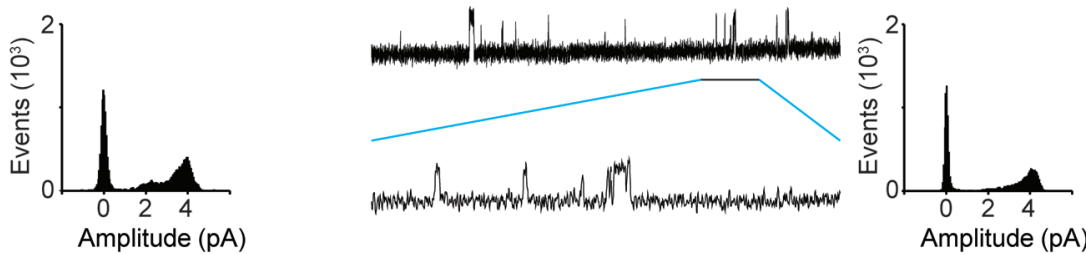

Amplitude (pA)

Figure 1. Cortex and hippocampal RyR2 channel remodeling result in the biochemical signature of leaky RyR2 in human HD brains. Representative SDSPAGE analysis and quantification (bands normalized to total RyR2) of RyR2 immunoprecipitated from cortex (A and B) and hippocampus (A and C) from human samples (see Supplemental Table 1). Control $(n=4)$; patients with HD $(n=4)$ (band intensities were normalized to total RyR2). (D) Single-channel recordings of RyR2 incorporated in planar lipid bilayers with $150 \mathrm{nM} \mathrm{Ca}{ }^{2+}$ in the cis chamber, corresponding to representative experiments performed with human cortex samples from controls and 3 patients with HD. RyR2 open probability ( $\mathrm{Po}$ ), mean open time (To) were increased, and the mean closed time (Tc) was decreased in HD RyR2 channels (E-G). $P_{0}$ was $0.014 \pm 0.005$ in control $(n=14)$ and in patients with HD increased to $0.095 \pm 0.01$ ( $n=13$ ). Results are expressed as mean \pm SD. Student's $t$ test; ${ }^{*} P<0.05$, control vs. HD patient. Cys-NO, cysteine nitrosylation; DNP, 2,4-dinitrophenylhydrazine.

(Figure 4A). There was also increased RyR2 PKA phosphorylation in C1 neurons, to a lesser extent than in the NA (Figure 4A). This finding was confirmed by immunoprecipitation. Brainstem RyR2 exhibited increased Ser2808 phosphorylation, oxidation, cysteine nitrosylation, and depletion of the stabilizing subunit calstabin2 (Figure 4, B and C). We treated the HD mice with S107 (blood-brain barrier [BBB] permeant) 


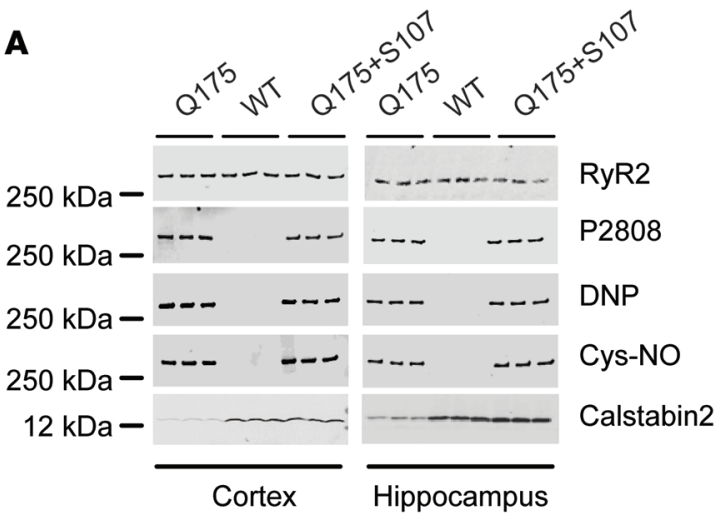

D

$[\mathrm{Ca}]_{\text {cyt }} 150 \mathrm{nM}$

WT cortex

Po 0.004 To 1.22 ms Tc $373.3 \mathrm{~ms}$

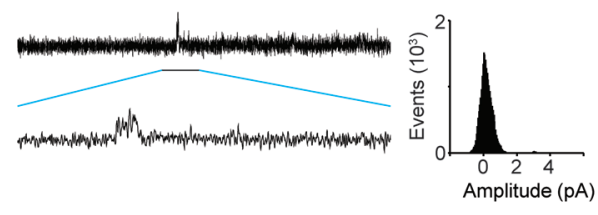

Q175 cortex + S107 (drinking water)

Po 0.008 To $2.16 \mathrm{~ms}$ Tc $98.75 \mathrm{~ms}$

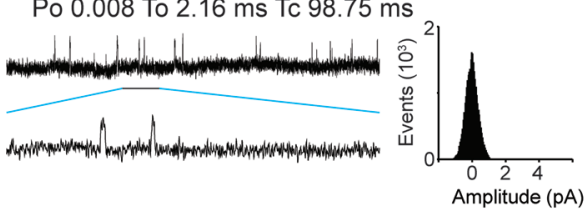

H

$[\mathrm{Ca}]_{\mathrm{cyt}} 150 \mathrm{nM}$

WT hippocampus

Po 0.005 To $2.89 \mathrm{~ms}$ Tc $462.3 \mathrm{~ms}$
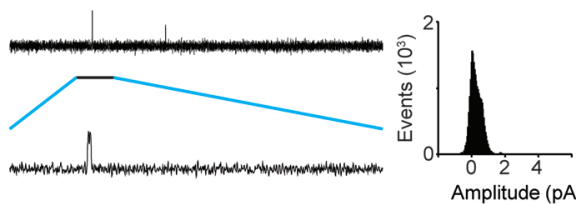

Q175 hippocampus + S107

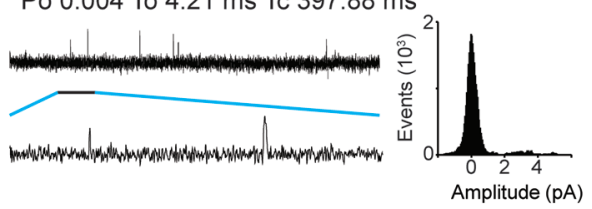

B

Mouse Cortex

口WT ロ Q175 ロQ175+S107

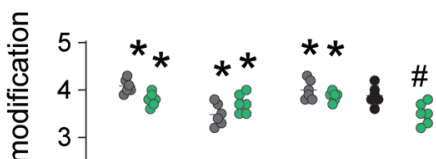

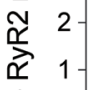

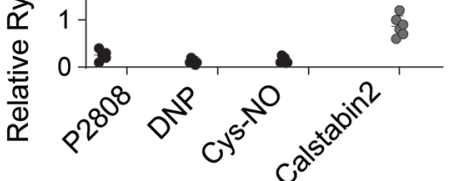

Q175 cortex

Po 0.182 To $7.96 \mathrm{~ms}$ Tc $42.71 \mathrm{~ms}$
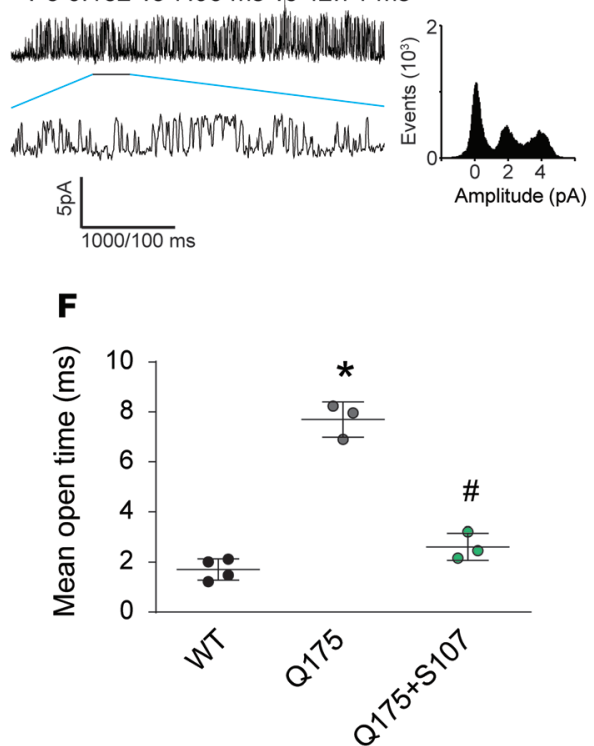

Q175 hippocampus Po 0.09 To 8.65 ms Tc 81.39 ms

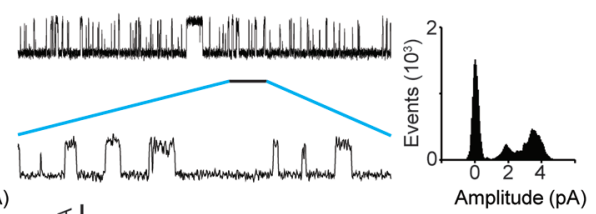

咅 $\frac{}{1000 / 100 \mathrm{~ms}}$

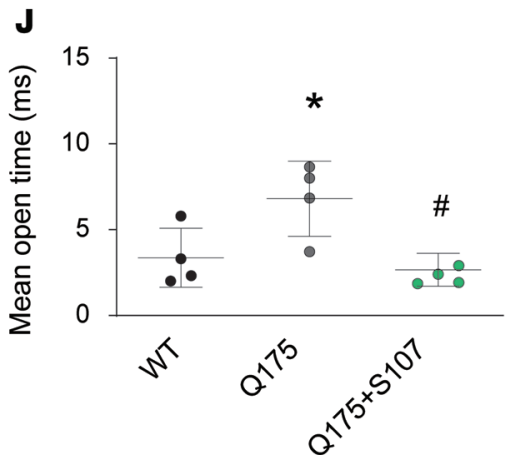

C Mouse Hippocampus

口 WT ロ Q175 ロQ175+S107
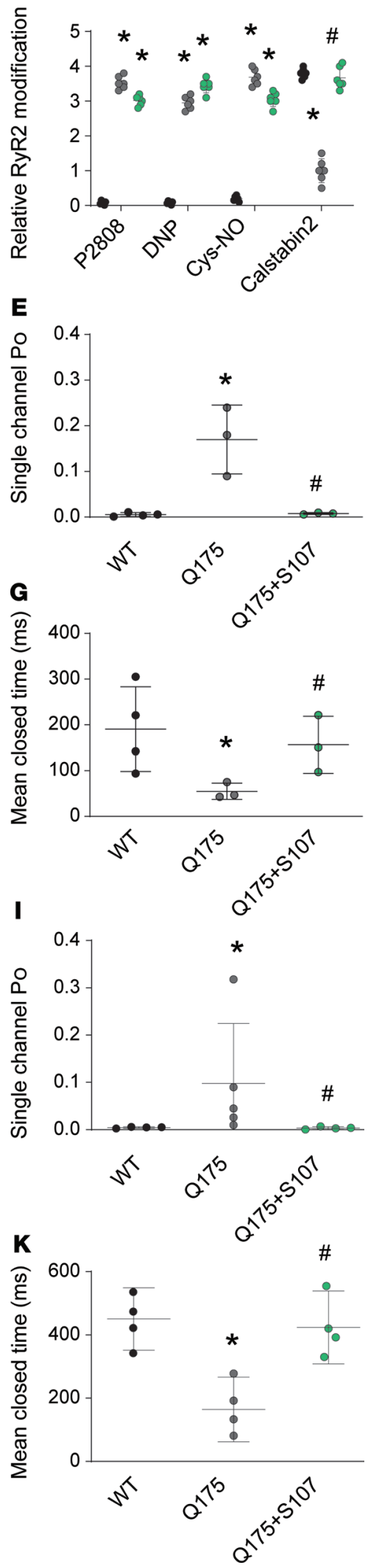
Figure 2. Cortical and hippocampal RyR2 channel remodeling results in the biochemical signature of leaky RyR2 in the brains of Q175 mice. Representative SDS-PAGE analysis and quantification of RyR2 immunoprecipitated from cortex (A and $\mathbf{B}$ ) and hippocampus (A and $\mathbf{C})$ from mouse samples. WT $(n=6)$, Q175 $(n=6)$, and Q175 $(n=6)$ treated with 5107 (bands' intensities were normalized to total RyR2). (D) Single-channel traces of RyR2 incorporated in planar lipid bilayers with $150 \mathrm{nM} \mathrm{Ca}^{2+}$ in the cis chamber, corresponding to representative experiments performed with mouse cortex samples from WT, Q175, and Q175 treated with S107. (E-G) RyR2 $\mathrm{P}_{0}$ was increased in HD. Mean $\mathrm{P}_{0}$ was $0.005 \pm 0.002$ in WT ( $\left.n=4\right)$ and in Q175 mice increased to $0.17 \pm 0.04(n=3)$ and restored by S107 treatment to $0.008 \pm 0.001(n=3)$. (H) Single-channel traces of RyR2 incorporated in planar lipid bilayers with $150 \mathrm{nM} \mathrm{Ca}{ }^{2+}$ in the cis chamber, corresponding to representative experiments performed with mouse hippocampus samples from WT, Q175, and Q175 treated with S107. RyR2 $P_{0}$ and $T_{0}$ were increased and Tc was decreased in HD (I-K). $P_{0}$ was $0.004 \pm 0.0007$ in WT ( $\left.n=4\right)$ and in Q175 mice increased to $0.09 \pm 0.05(n=4)$ and restored by $S 107$ treatment to $0.003 \pm 0.001(n=4)$. Data (mean \pm SD) analysis was performed by 1 -way ANOVA. Bonferroni's posttest revealed ${ }^{*} P<0.05$ vs. WT, ${ }^{*} P<0.05$ vs. Q175.

and ARM036 (BBB nonpermeant) Rycals to understand the link between brainstem RyR2 abnormalities and autonomic dysfunction. S107 treatment, but not ARM036, prevented calstabin2 dissociation from the RyR2 macromolecular complex in the brainstem, demonstrating a central origin of these defects. In addition, cardiac RyR2 exhibited increased Ser2808 phosphorylation, oxidation, cysteine nitrosylation, and depletion of the stabilizing subunit calstabin2 (Figure 5, A and B). This cardiac RyR2 remodeling was associated with a significant increase in RyR2 $\mathrm{P}_{\mathrm{O}}$ in heart samples from Q175 mice consistent with leaky channels (Figure 5, C-F). Both S107 and ARM036 prevented calstabin2 dissociation from cardiac RyR2.

Remodeled RyR2 in brainstems contributes to altered HRV and cardiac arrhythmias in a murine model of $H D$. To evaluate the ANS outflows, we monitored cardiac electric activity (ECG) using a telemetric system over 24 hours in freely moving, conscious WT, Q175, and Q175 animals treated with S107 or ARM036. Heart rate $(\mathrm{HR})$ is controlled by the activity of the pacemaker and modulated by the sympathetic and parasympathetic limbs of the ANS. We hypothesized that the RyR2 remodeling in NA and C1 neurons could induce a sympathovagal imbalance contributing to cardiac arrhythmias. During the awake period, we observed a slight but not significant increase in HR in Q175 mice (Figure 6, A and B). During the rest period, HR was significantly increased in Q175 mice compared with WT, suggesting a reduction in the parasympathetic tone during rest in line with the NA and RyR2 remodeling observed in the brainstem slices. Moreover, fixing the calcium leak with S107 but not ARM036 significantly reduced HR at rest (Figure 6, A and B).

Hexamethonium, which blocks both sympathetic and parasympathetic limbs of the ANS (74), caused a significant reduction in HR in both Q175 and Q175+ARM036 mice. These data suggest that the increased HR observed in Q175 mice was driven by decreased parasympathetic activity and/or increased sympathetic activity (Figure 6C).

We then analyzed the activity of the ANS manifested by beat-to-beat HRV (Figure 6, D-I). The low-frequency bands reflect mostly sympathetic modulation of heart rhythm, and oscillations in high-frequency bands reflect exclusively parasympathetic vagal activity. Thus, the low frequency/high frequency ratio reflects sympathovagal balance (75). The low frequency was similar between all the groups during active and rest periods, suggesting that sympathetic regulation of cardiac activity was normal in the Q175 HD mouse model. In contrast, there was a significant decrease in the high frequency during the rest period in Q175 mice compared with WT consistent with impaired parasympathetic activity. Q175 mice exhibited a lower low frequency/high frequency ratio compared with WT during the active period and a higher low frequency/high frequency ratio during the rest period (Figure 6, H and I). These data suggest that RyR2-mediated ER $\mathrm{Ca}^{2+}$ leak in the brainstem alters sympathovagal balance, through a reduction in parasympathetic activity that may promote cardiac arrhythmias. Indeed, ventricular extrasystoles (VESs) were increased in Q175 mice during the rest period but did not change during the awake period. The increased number of extrasystoles was abolished by both S107 and ARM036, suggesting that these VESs were triggered in the heart by diastolic SR Ca ${ }^{2+}$ leak as previously described (76). The remodeling of RyR2 in the heart described above accounts for diastolic SR $\mathrm{Ca}^{2+}$ leak and subsequent VES (Figure 6, J and K). Next, we evaluated cardiac function using ECG to measure the left ventricular ejection fraction (LVEF) and speckle tracking imaging that allows earlier detection of left ventricular systolic dysfunction, a strong predictor of heart failure and mortality (77). The LVEF in Q175 mice was normal; however, the left ventricular global longitudinal strain was reduced, indicating a predisposition to heart failure (Supplemental Figure 5, A-C).

Remodeled leaky RyR2 in brainstems contributes to respiratory dysfunction. Respiratory dysfunction including chest muscle rigidity, respiratory muscle weakness, difficulty in clearing airway secretions, and swallowing abnormalities have been reported in patients with neurodegenerative disorders, including $\mathrm{HD}(9,10)$. 


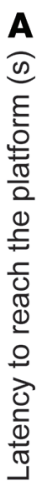

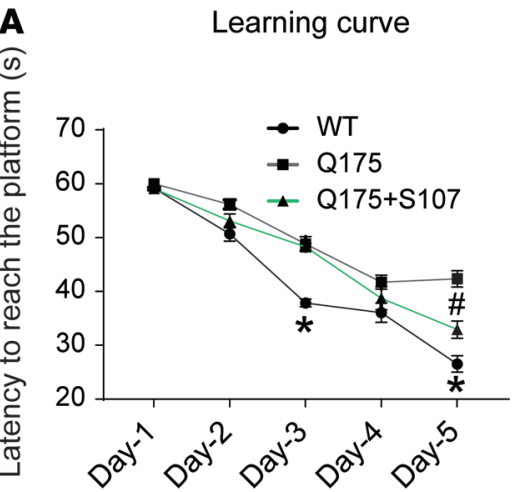

Training (day)
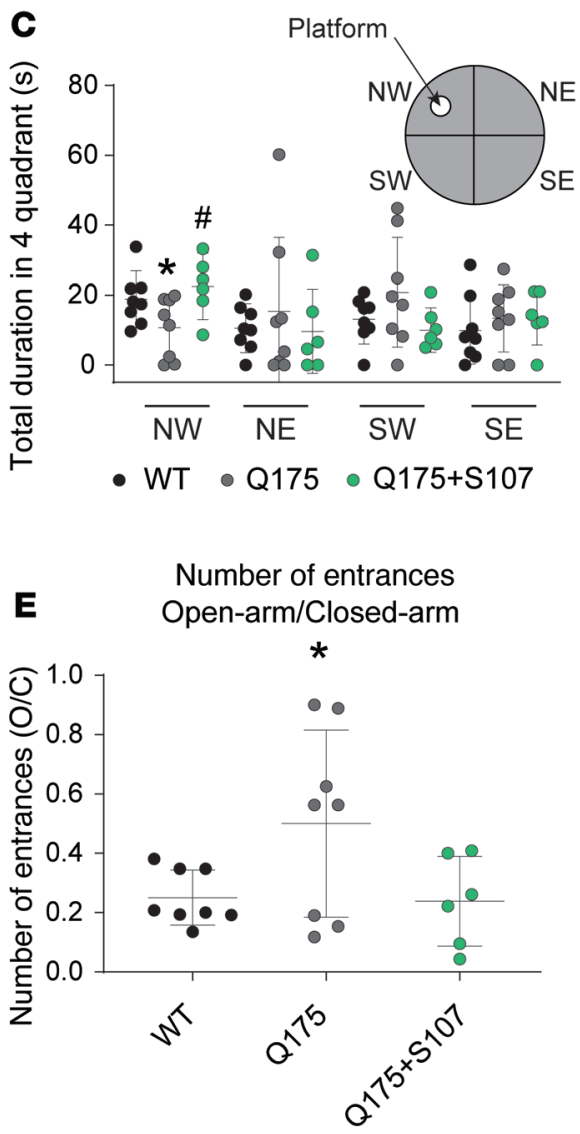

B

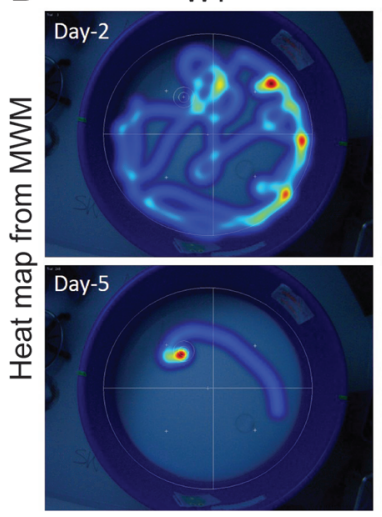

Q175+S107

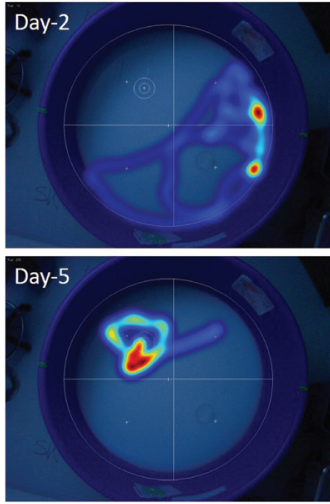

D Target crossings in probe trial
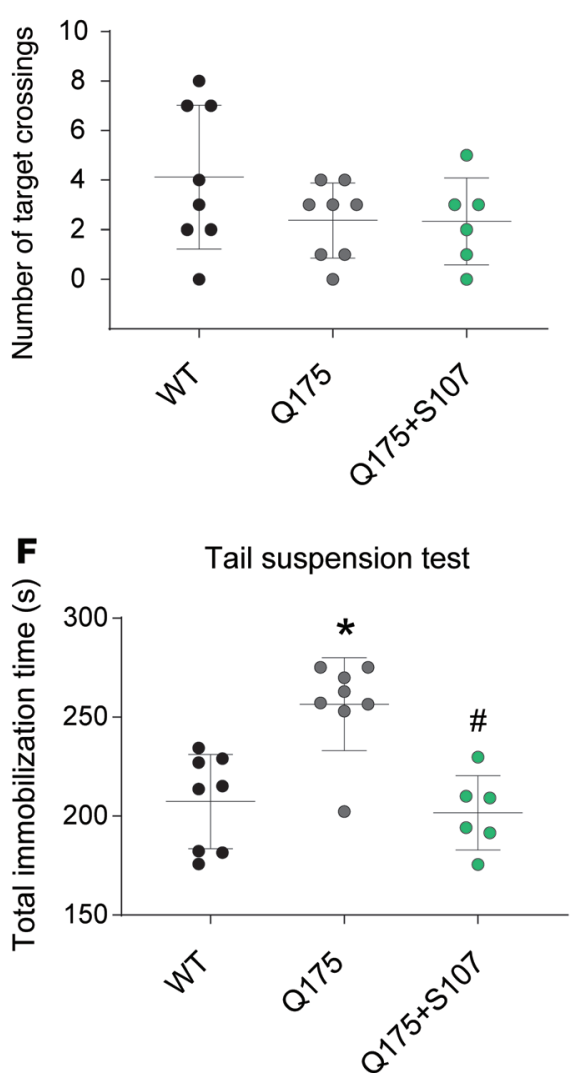

Figure 3. Long-term learning and memory deficits in Q175 mice. S107 treatment targeting RyR2 channels improves cognitive function in HD. (A) Learning curves showing the escape latency during a 5-day training period in the Morris water maze (MWM). (B) The heatmaps from all the training trials were recorded (Noldus Information Technology Inc.) and downloaded. Representative heatmaps from WT (left column), Q175 (middle column), and S107-treated Q175 (right column) on day 2 (upper panels) and day 5 (lower panels) are shown. (C) The time spent in all quadrants. (D) The number of target crossings on day 6 probe trial of the MWM. (E) The ratio of the number of entries to open arms versus closed arms of the elevated plus maze (EPM). (F) Total immobilization time of mice during 300 seconds of tail suspension test. The same groups of mice were used for MWM, EPM, and tail suspension. WT ( $n=8)$, Q175 ( $n=8$ ), and $\mathrm{Q} 175+\mathrm{S} 107(n=6)$. Data (mean \pm SD) analysis was performed by 1-way ANOVA. Bonferroni's posttest revealed ${ }^{*} P<0.05$ vs. WT, ${ }^{*} P<0.05$ vs. $Q 175$.

We evaluated ex vivo and in vivo the respiratory function in Q175 HD mice. In order to distinguish between centrally mediated versus peripherally mediated effects on respiratory function, we used 2 Rycal drugs that fix leaky RyR channels, S107 and ARM036.

To evaluate the intrinsic properties of the respiratory muscle, we compared the ex vivo diaphragmatic function in Q175 versus WT mice. Force production was significantly reduced (Figure 7, A-E) 
A

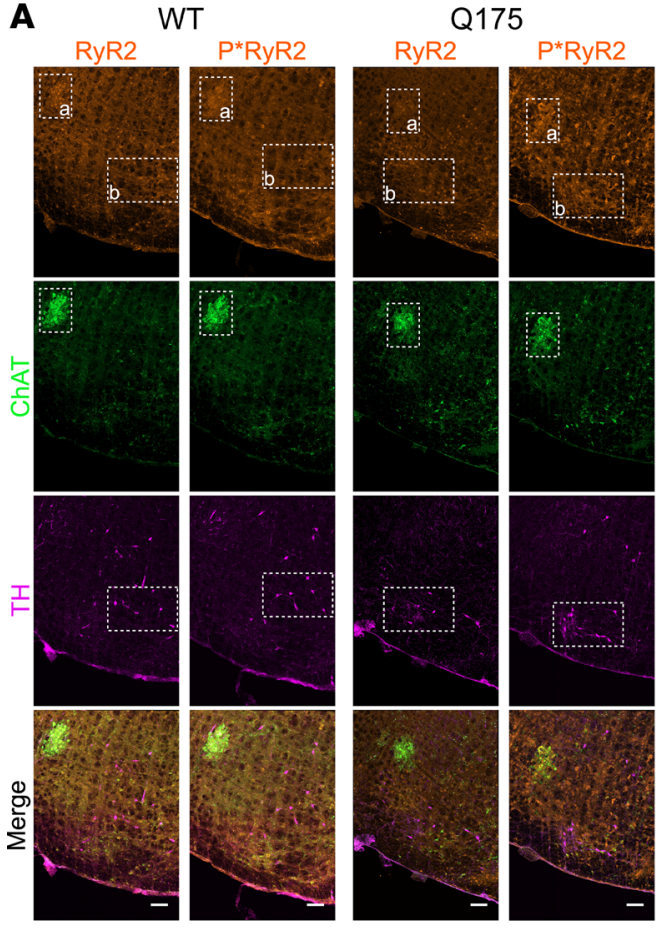

WT

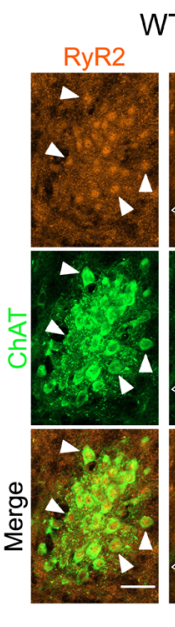

WT

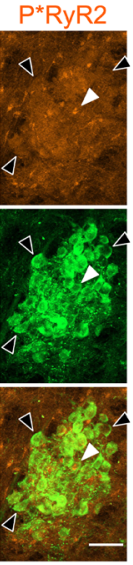

Q175

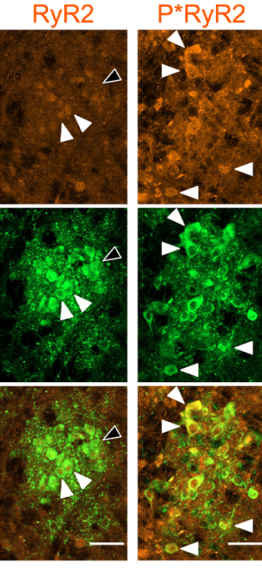

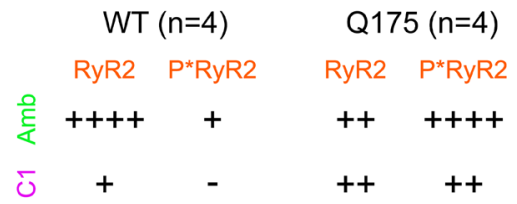
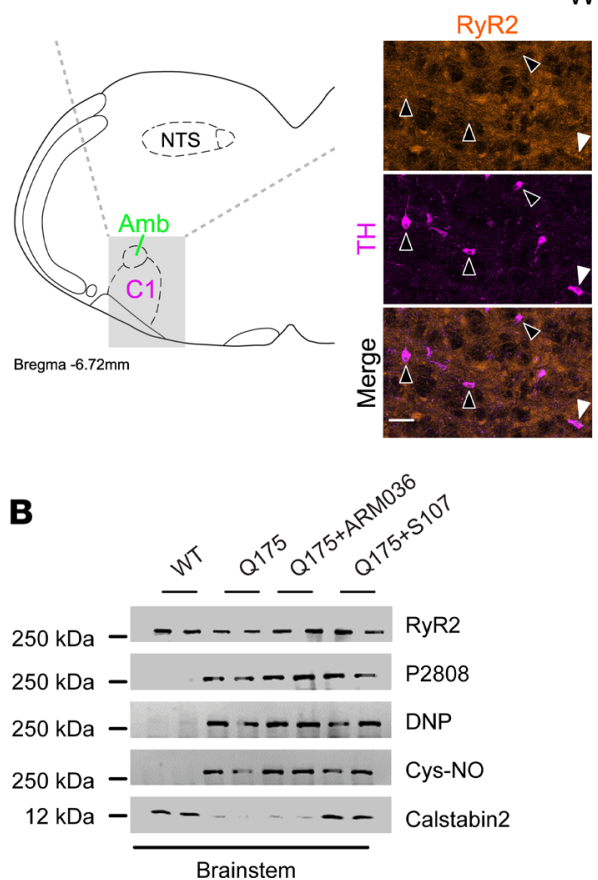

Q175
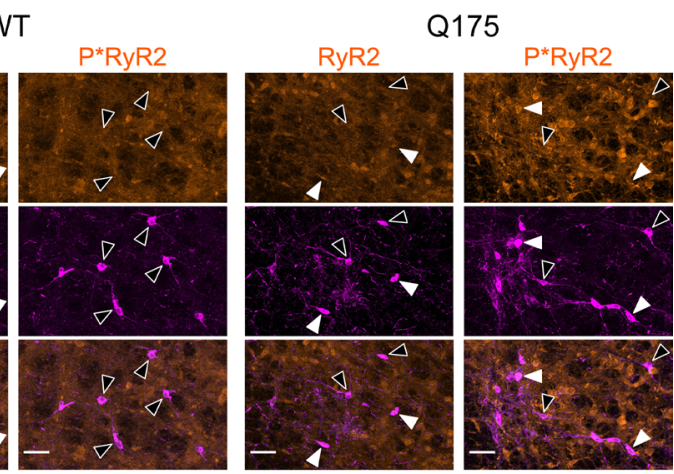

- Q175+ARM036

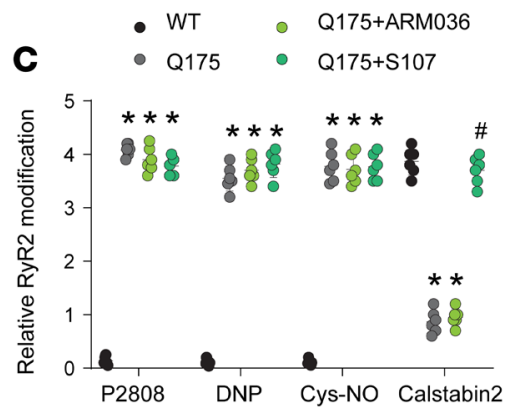

Figure 4. Brainstem RyR2 remodeling induces NA disruption. (A) Immunostaining and semiquantitative analysis of total and phosphorylated RyR2 ( ${ }^{*}$ RyR2) on Ser2808 in NA neurons (choline acetyltransferase positive, ChAT positive), which include cardiac parasympathetic neurons, and in presympathetic rostral ventrolateral medulla C1 neurons (tyrosine hydroxylase positive, TH positive) in the brainstems of WT and Q175 mice. White arrows show ChAT/TH neurons with RyR2/P*RyR2 colocalization; black arrows show ChAT/TH neurons without RyR2/P*RyR2 colocalization. Semiquantitative analysis of ChAT/TH and RyR2/ $P *$ RyR2 colocalization were made as follows: - $=0 \%$ costaining, $+=1 \%$ to $25 \%$ costaining, $++=26 \%$ to $50 \%$ costaining, $+++=51 \%$ to $75 \%$ costaining, and ++++ $=76 \%$ to $100 \%$ costaining. Analysis was made in 4 WT and 4 Q175 mice, bilaterally in $2-3$ sections per animal. (B and $\mathbf{C}$ ) Representative SDS-PAGE analysis and quantification of RyR2 immunoprecipitated from the brainstems collected from WT $(n=6)$, Q175 $(n=6)$, Q175+ARM036 $(n=6)$, and Q175+S107 $(n=6)$ mice (band intensities were normalized to total RyR2). Data (mean $\pm S D$ ) analysis was performed by 1-way ANOVA. Bonferroni's posttest revealed ${ }^{*} P<0.05 \mathrm{vs.} \mathrm{WT,}{ }^{*} P$ $<0.05$ vs. Q175. Scale bars: $100 \mu \mathrm{m}$ (top left), $50 \mu \mathrm{m}$ (top right and middle right). Amb, nucleus ambiguus; NTS, nucleus tractus solitarius.

at different stimulation frequencies $(P<0.05)$ in Q175 mice compared with their control littermates. Both S107 and ARM036 treatments restored diaphragmatic force generation in Q175 mice (Figure 7, C-E). Interestingly, extensor digitorum longus (EDL) and soleus contractile function were not affected in HD mice (Supplemental Figure 5, H-Q), suggesting that the muscle dysfunction was more severe in the diaphragm. One possibility is that the severity of the muscle dysfunction in the diaphragm was due to defective central regulation of respiratory function.

We used whole-body plethysmography to compare the respiratory function of Q175 versus WT mice with room air or hypercapnia $\left(8 \% \mathrm{CO}_{2}\right)($ Figure $7, \mathrm{~F}-\mathrm{H})$. With room air WT and Q175 mice had equivalent tidal volumes (TVs), respiratory frequency $(\mathrm{F})$, minute ventilation $(\mathrm{MV})$, and inspiration and expiration times (Figure 7, F-H; and Supplemental Figure 5, P-R). With hypercapnia $\left(8 \% \mathrm{CO}_{2}\right)$, 
A

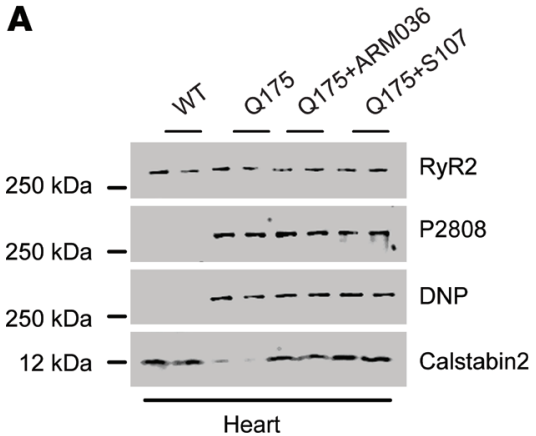

C

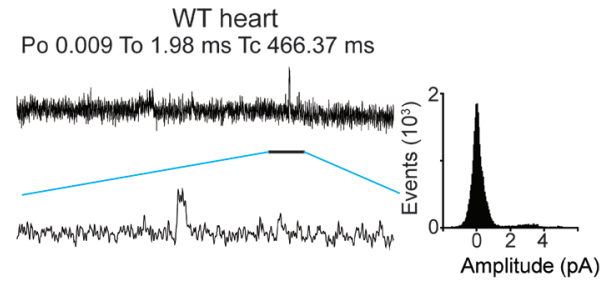

Q175 heart + S107

Po 0.008 To $2.82 \mathrm{~ms}$ Tc $229.74 \mathrm{~ms}$

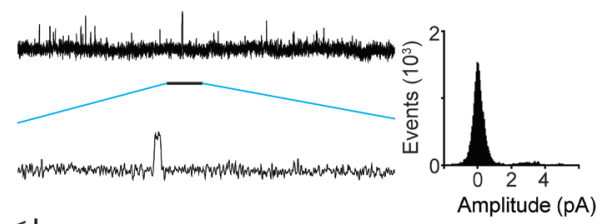

离 $1000 / 100 \mathrm{~ms}$

D

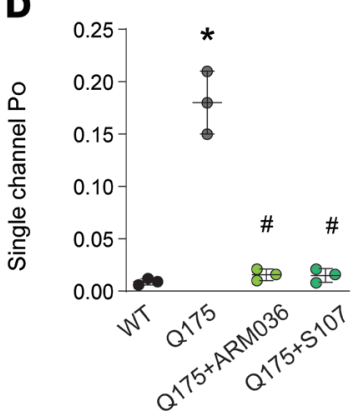

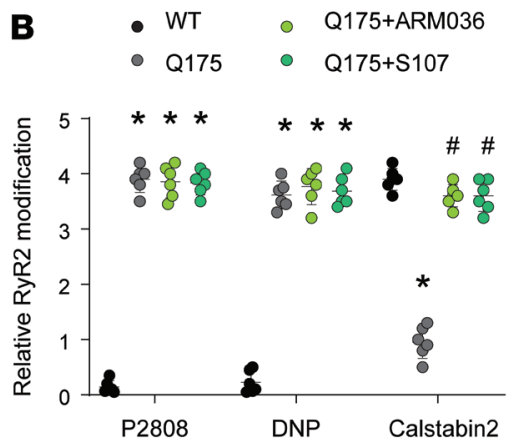

Q175 hear Po 0.21 To $8.32 \mathrm{~ms}$ Tc $55.64 \mathrm{~ms}$

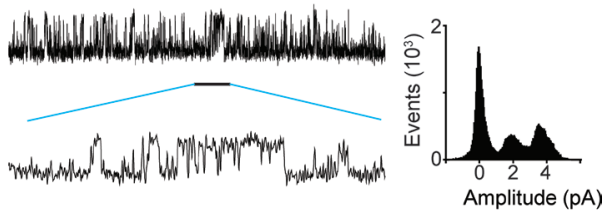

Q175 heart + ARM036

Po 0.01 To $3.41 \mathrm{~ms}$ Tc $305.13 \mathrm{~ms}$

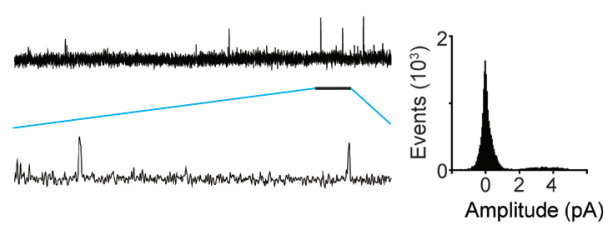

Figure 5. Cardiac RyR2 remodeling in Q175 mice. (A and B) Representative SDS-PAGE analysis and quantification of RyR2 immunoprecipitated from the heart samples from WT $(n=6)$, Q175 $(n=6)$, Q175+ARM036 $(n=6)$, and Q175+S107 $(n=6)$ (bands' intensities were normalized to total RyR2). (C) Single-channel traces of RyR2 incorporated in planar lipid bilayers with $150 \mathrm{nM} \mathrm{Ca}{ }^{2+}$ in the cis chamber, corresponding to representative experiments performed with heart samples from WT, Q175, Q175+ARM036, and Q175+S107 mice. (D-F) Single-channel analysis: $P_{0}$ was $0.009 \pm 0.001$ in WT $(n=3)$ and in Q175 mice increased to $0.18 \pm 0.01(n=3)$ and restored by 5107 treatment to $0.015 \pm 0.003(n=3)$ and ARM036 to $0.015 \pm 0.003(n=3)$. Data (mean \pm SD) analysis was performed by 1-way ANOVA. Bonferroni's posttest revealed ${ }^{*} P<0.05$ vs. WT, ${ }^{*} P<0.05$ vs. Q175.

Q175 mice exhibited significantly reduced MV compared with the WT mice and decreased TV $(P<$ 0.05 ) (Figure 7, $\mathrm{F}$ and $\mathrm{H}$ ).

S107 and ARM036 administration had no effect on respiratory function in mice breathing room air (Figure 7, F-H). In contrast, S107 treatment, but not ARM036, rescued respiratory function in Q175 mice under hypercapnia. These data suggest that leaky neuronal RyR2 alters central respiratory control in Q175 HD mice. In addition, voluntary activity as determined using running wheel was significantly reduced in terms of time spent on the wheel in Q175 mice compared with control without any difference in the mean 
A

WT

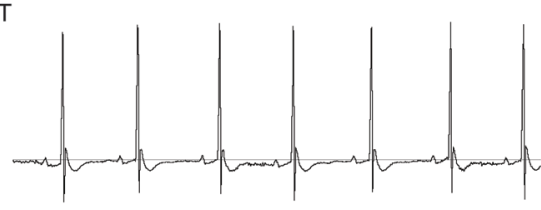

Q175

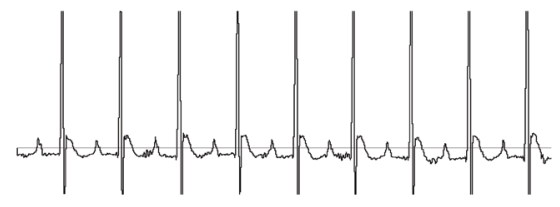

Q175+ARM036

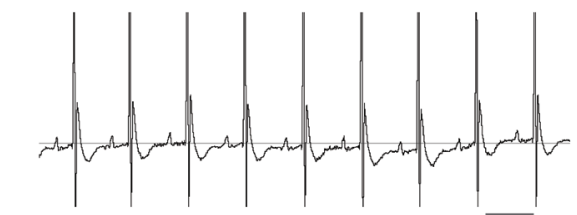

Q175+S107

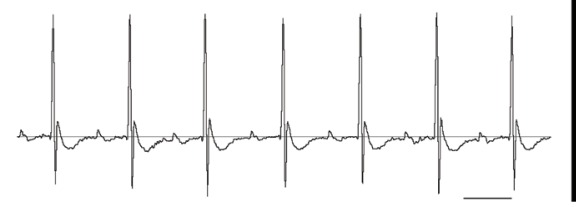

Awake Period
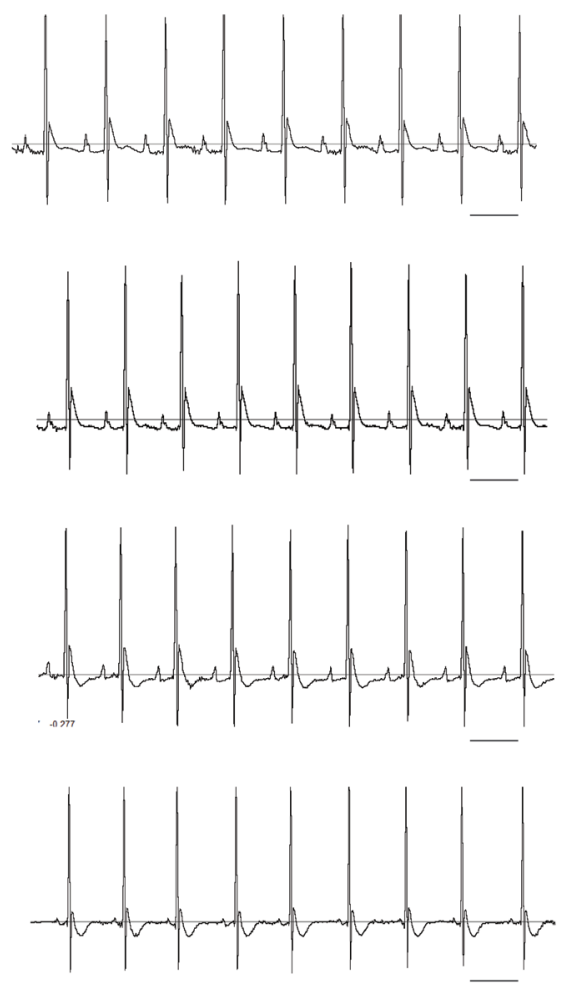

B $\quad$ Rest Period

- Awake Period

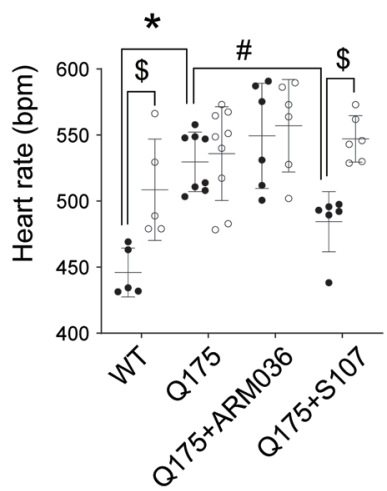

C Baseline

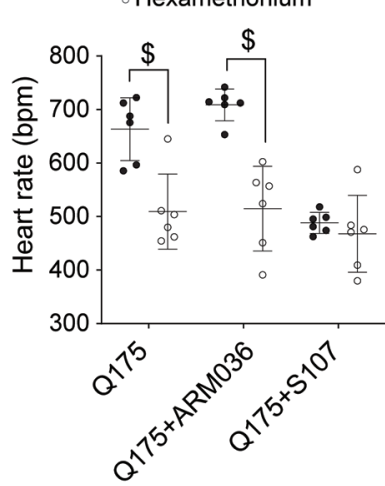

D Awake Period $\quad$ E $\quad$ Rest Period F Awake Period

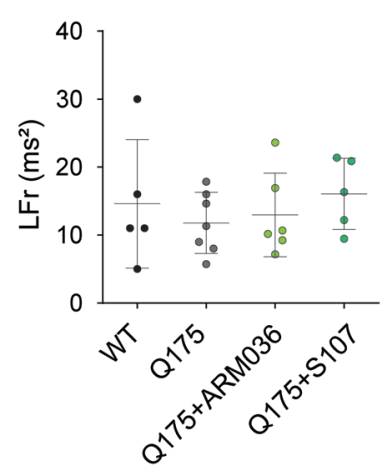

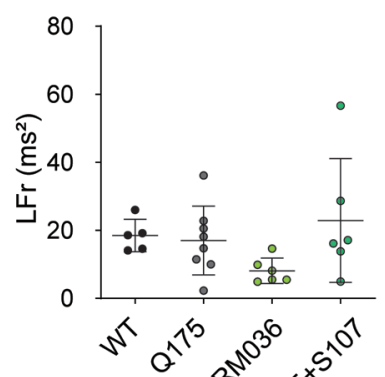

$0^{10^{x p^{25}}} 0^{n^{t^{x^{5}}}}$

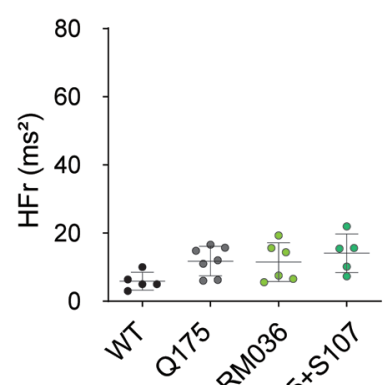

$0^{n^{x^{x+2}}} 0^{\lambda^{1 x^{2}}}$
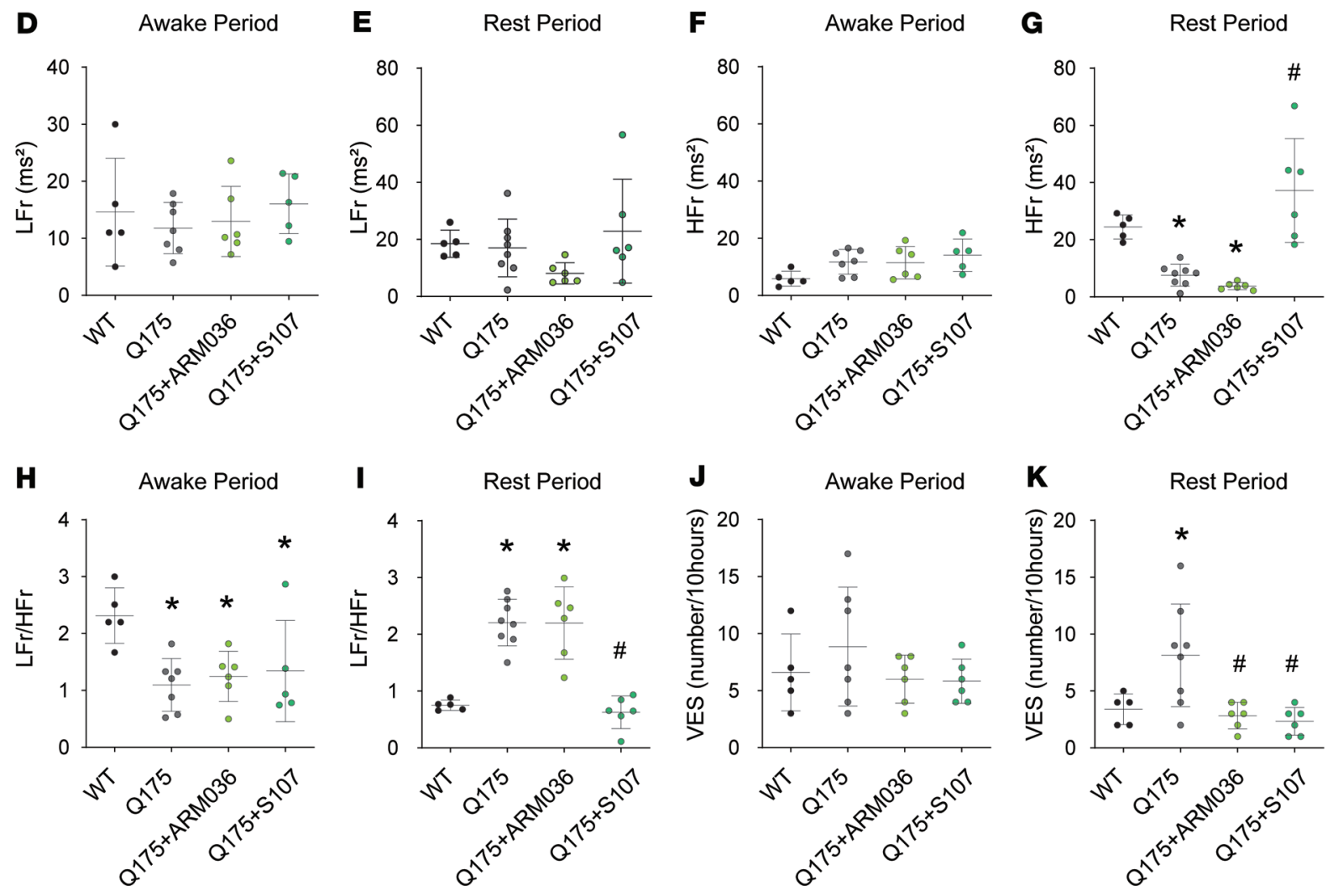
Figure 6. Sympathovagal imbalance and arrhythmias in HD during rest period. (A) Representative ECC trace in freely moving conscious animals allowing HR (bpm) measurement in WT and Q175 mice treated or not with S107 or ARM036 during light (rest period) and dark (awake period) cycles. (B) HR (bpm) average during awake and rest periods in WT $(n=5)$, Q175 $(n=8)$, and Q175 mice treated or not with S107 $(n=6)$ or ARM036 $(n=6)$. (C) HR average record. Hexamethonium injection ( $20 \mathrm{mg} / \mathrm{kg}$ ) had moderate effects on HR related to suppression of cardiac autonomic control of HR, that is, lowering HR during the daylight period in Q175 $(n=6)$ and Q175 mice treated or not with S107 $(n=6)$ or ARM036 $(n=6)$. Data (mean \pm SD) analysis was performed by 1-way ANOVA. Bonferroni's posttest revealed ${ }^{*} P<0.05$ vs. WT, ${ }^{*} P<0.05$ vs. Q175 at rest period. Student's $t$ test, ${ }^{\$} P<0.05$ rest vs. awake period and baseline vs. hexamethonium. ( $\mathbf{D}$ and $\mathbf{E}$ ) Low-frequency (LFr) spectral power density measured by HRV analysis using fast Fourier transformation (LFr: 0.15-1.5 Hz) in WT, Q175, Q175+ARM036, and Q175+S107 during rest and awake periods ( $n=5-8$ mouse/group). (F and G) High-frequency (HFr) spectral power measured by HRV analysis using fast Fourier transformation (HFr: 1.5-5 Hz) during rest and awake periods in WT, Q175, Q175+ARM036, and Q175+S107 ( $n=5-8$ mouse/group). ( $\mathbf{H}$ and $\mathbf{I}$ ) LFr/HFr ratio ( $n=5-8$ mouse/group). ( $\mathbf{J}$ and $\mathbf{K}$ ) Number of isolated and triplet (3 consecutive) ventricular extrasystoles (VESs) during 10 hours in WT, Q175, Q175+ARM036, and Q175+S107 during rest and awake periods ( $n=5-8$ mouse/group). Comparison of HRV (standard deviation of NN intervals) and VESs at rest versus awake is shown in Supplemental Figure 5, D-F. Representative example of VESs in Q175 mice is shown in Supplemental Figure 5G. Data (mean \pm SD) analysis was performed by 1-way ANOVA. Bonferroni's posttest revealed ${ }^{*} P<0.05$ vs. WT, ${ }^{\#} P<0.05$ vs. Q175. Scale bar: 110 ms.

speed (Figure 7, I-K). Both S107 and ARM036 treatments similarly rescued the decreased voluntary activity observed in our HD mice.

Diaphragmatic dysfunction in HD is associated with impaired excitation contraction coupling. Diaphragmatic dysfunction can occur in response to increased beta-adrenergic signaling, which causes PKA-mediated RyR1 hyperphosphorylation and leaky channels (78). RyR1 Ser2844 phosphorylation, oxidation, and cysteine nitrosylation were increased and calstabin1 was depleted from RyR1 in Q175 diaphragms (Figure 8, A and B). RyR1 remodeling was associated with a significant increase in RyR1 $\mathrm{P}_{\mathrm{O}}$ under nonactivating conditions $\left(\left[\mathrm{Ca}^{2+}\right]_{\mathrm{cyt}}=150 \mathrm{nM}\right)$, consistent with leaky channels, which were fixed by treatment with either S107 or ARM036 (Figure 8, C-F). Moreover, diaphragmatic dysfunction was not due to reduced cross-sectional area (Figure $8, \mathrm{G}$ and $\mathrm{H})$.

\section{Discussion}

Calcium is a second messenger that regulates activity of numerous cellular processes, including activation of protein kinases and phosphatases, proteases, ion transporters and channels, neurotransmitter vesicle release, and gene transcription. $\mathrm{Ca}^{2+}$ is stored in the endo/sarcoplasmic reticulum compartment and can be released via IP3Rs and/or RyRs $(47,79)$. In pathological conditions, remodeled RyR channels have been shown to mediate ER/SR Ca ${ }^{2+}$ leak into the cytosol at rest, leading to local increases of $\left[\mathrm{Ca}^{2+}\right]_{\text {cyt }}$ and activation of pathological signals (60). In neurons, such increases in $\left[\mathrm{Ca}^{2+}\right]_{\text {cyt }}$ can alter synaptic plasticity, survival/growth, and other essential signals (80). Interestingly, it has been shown in $\mathrm{R} 6 / 2 \mathrm{HD}$ mice that increased $\left[\mathrm{Ca}^{2+}\right]_{\mathrm{cyt}}$ is linked to locomotor dysfunction (81).

In the present study we show that RyR2 channels in the brain are oxidized, PKA phosphorylated, nitrosylated, and depleted of the stabilizing subunit calstabin in both human patients with HD and in 3 models of $\mathrm{HD}$ (R6/2, Q111, and Q175). This biochemical signature of leaky RyR2 is consistent with previous reports of increased $\left[\mathrm{Ca}^{2+}\right]_{\text {cyt }}$ in $\operatorname{HD}$ neurons $(37,81)$. Moreover, the observation that fixing RyR2-mediated $\mathrm{ER} \mathrm{Ca}^{2+}$ leak with S107 improves cognitive and locomotor function in a murine model of HD (Q175 mice) suggests that leaky RyR2 play a heretofore crucial role in HD pathogenesis. This result is consistent with the in vitro neuroprotection and improved motor function previously reported using dantrolene in YAC128 mice $(66,80)$. Indeed, increased cytosolic $\mathrm{Ca}^{2+}$ concentrations can activate $\mathrm{Ca}^{2+}$-dependent kinases, such as CaMKII, which may contribute to further RyR2 remodeling and $\mathrm{Ca}^{2+}$ leak (82). Activation of CaMKII has been reported in cardiac tissues, cortex, and striatum of BACHD and R6/2 murine models of HD, where the expression of CaMKIV was increased $(83,84)$. Of note, HD likely shares common features with $\mathrm{AD}$, in which enhanced RyR-mediated $\mathrm{ER} \mathrm{Ca}^{2+}$ leak has been linked to pathological posttranslational modifications (e.g., PKA phosphorylation, oxidation/nitrosylation, and calstabin depletion) (58).

While the mechanisms involved in HD remain unclear, several hypotheses have been put forward: mutated huntingtin aggregates form inclusion bodies inside neurons, and insoluble huntingtin causes mitochondrial dysfunction, as well as $\mathrm{Ca}^{2+}$ dyshomeostasis and defective protein-protein interactions and vesicular transport of proteins including neurotransmitter receptors, ultimately leading to neuronal death. Moreover, mutated huntingtin microaggregates increase ROS production in the brain (85). Here we show increased ROS production in Q175 brains and increased RyR2 oxidation, which causes ER Ca ${ }^{2+}$ leak (Supplemental Figure 3A).

Widespread pathology beyond the brain in HD. In addition to the classic symptoms, HD is complicated by weight loss, heart failure, and respiratory and swallowing muscle dysfunction. These features can appear early in the disease course and can eventually contribute substantially to both morbidity and mortality (4). 
A
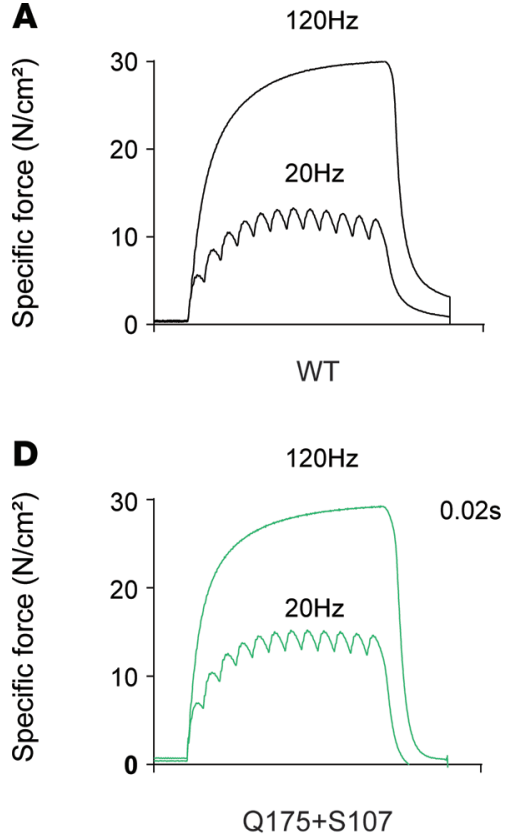

B

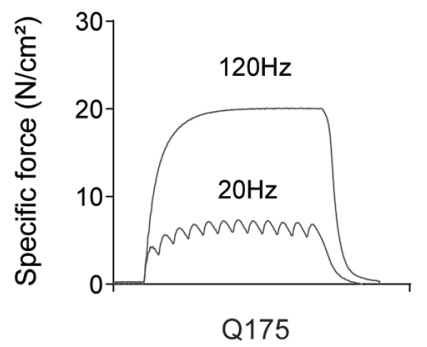

$\mathbf{E}$

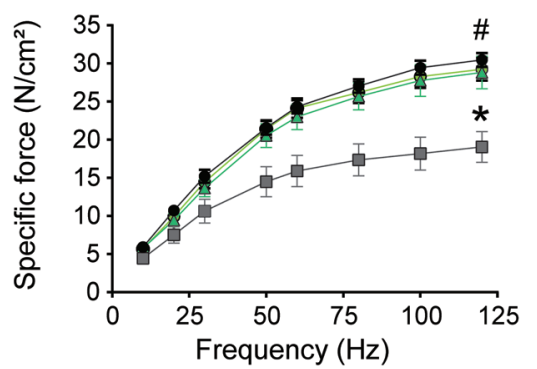

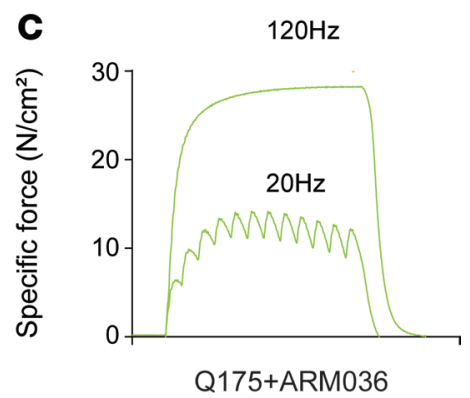

- WT

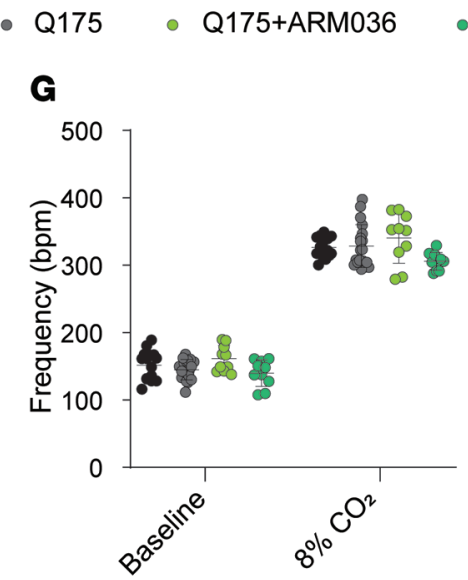

Q175+S107
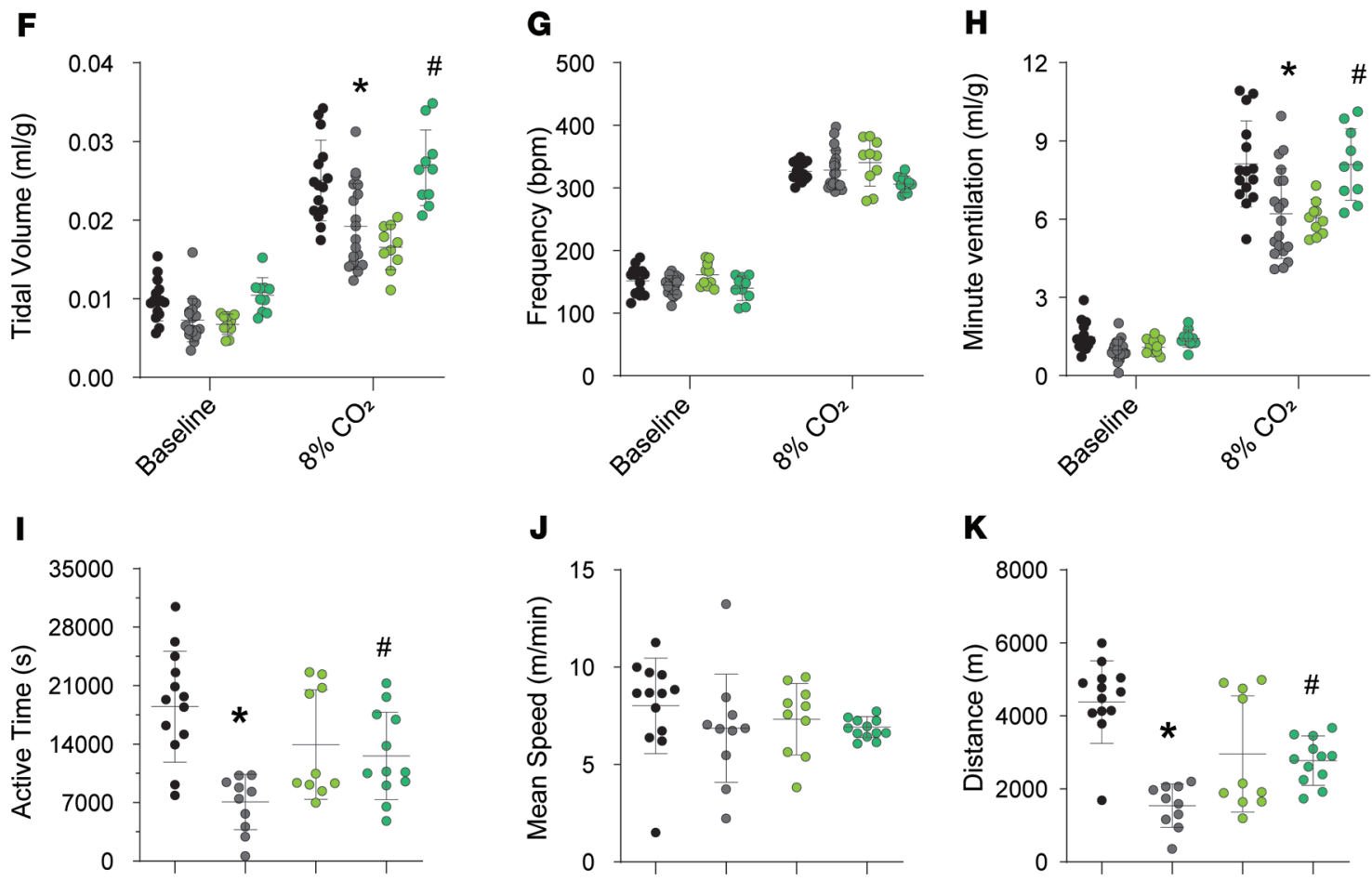

Figure 7. RyR-mediated SR Ca ${ }^{2+}$ leak contributes to respiratory dysfunction and reduces voluntary activity in a murine model of HD. (A-D) Representative records of diaphragmatic specific force production measured ex vivo at 20 and $120 \mathrm{~Hz}$ in muscle bundles under isometric conditions in WT, Q175, Q175+ARM036, and Q175+S107 mice. (E) Average force-frequency relationship recorded in WT $(n=13)$, Q175 $(n=10)$, Q175+ARM036 $(n=9)$, and Q175+S107 $(n=8)$ mice. Data (mean \pm SEM) analysis was performed by 2 -way ANOVA. Bonferroni's posttest revealed ${ }^{*} P<0.05$ vs. WT, ${ }^{\#}<<0.05$ Q175 vs. Q175+S107/ ARM036. (F) Tidal volume $(\mathrm{mL} / \mathrm{g})$ at rest and during $\mathrm{CO}_{2}$ stimulation. (C) Respiratory frequency $(\mathrm{bpm})$ at rest and during $\mathrm{CO}_{2}$ stimulation $(\mathbf{H})$ Minute volume $(\mathrm{mL} / \mathrm{g})$ at rest and during $\mathrm{CO}_{2}$ stimulation in WT $(n=15)$, Q175 $(n=19)$, Q175+ARMO36 $(n=10)$, and Q175+S107 $(n=10)$. The CO ${ }_{2}$ values were recorded 10 minutes after the initiation of $\mathrm{CO}_{2}$ stimulation. Data (mean $\pm \mathrm{SD}$ ) analysis was performed by 1-way ANOVA. Bonferroni's posttest revealed ${ }^{*} P<0.05$ vs. $W T$, ${ }^{P} P<0.05$ vs. Q175. (I-K) Active time (seconds), mean speed $(\mathrm{m} / \mathrm{min})$, and running distance $(\mathrm{m})$ for voluntary activity on running wheels, respectively, in WT ( $n=13)$, Q175 $(n=10)$, Q175+ARM036 $(n=10)$, and Q175+S107 mice $(n=12)$. Data (mean \pm SD) analysis was performed by 1-way ANOVA. Bonferroni's posttest revealed ${ }^{*} P<0.05$ vs. WT, ${ }^{*} P<0.05$ vs. Q175. 
A

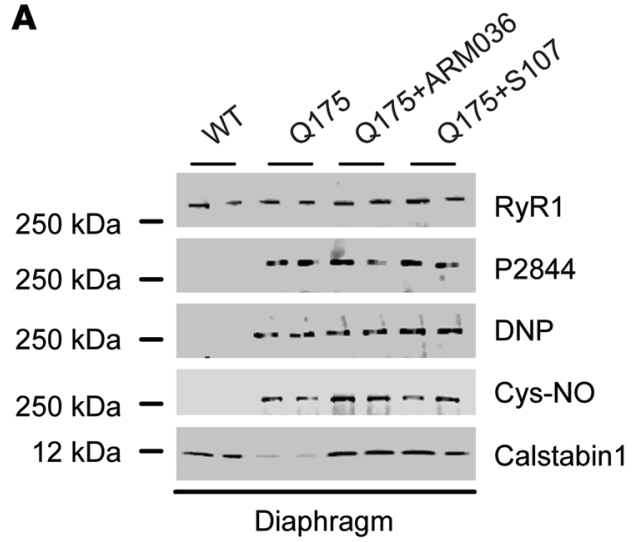

B $\quad W T: Q 175+A R M 036$

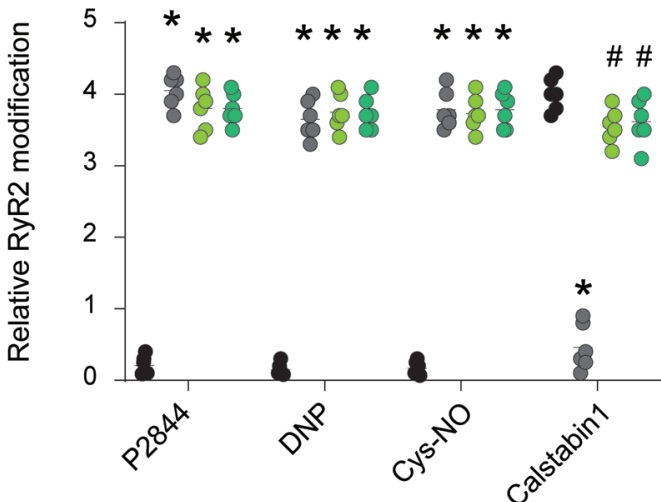

$[\mathrm{Ca}]_{\mathrm{cyt}} 150 \mathrm{nM}$

WT diaphragm

Po 0.006 To $1.89 \mathrm{~ms}$ Tc $484.2 \mathrm{~ms}$

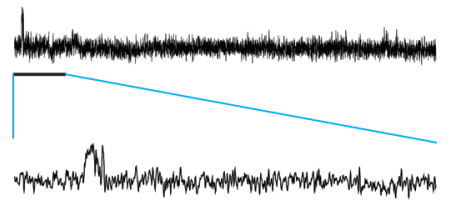

Q175 diaphragm + S107

Po 0.01 To $3.31 \mathrm{~ms}$ Tc $212.5 \mathrm{~ms}$

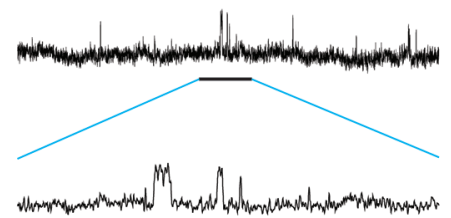

D

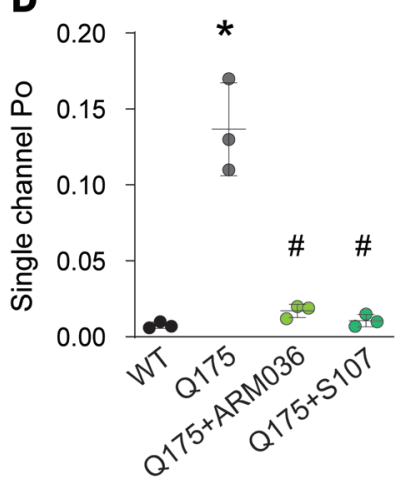

F

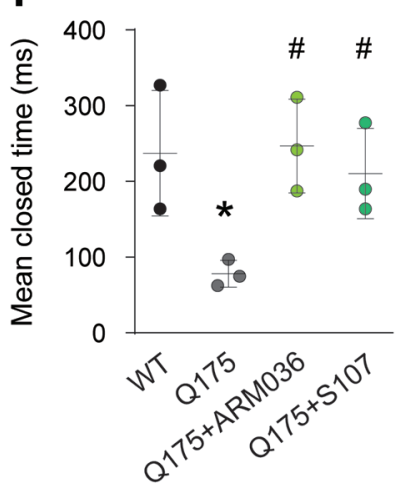

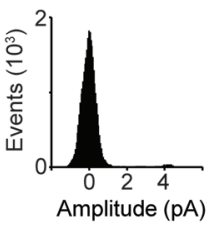

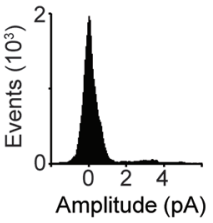

E

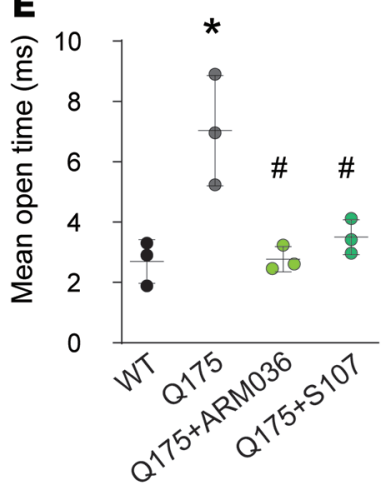

G

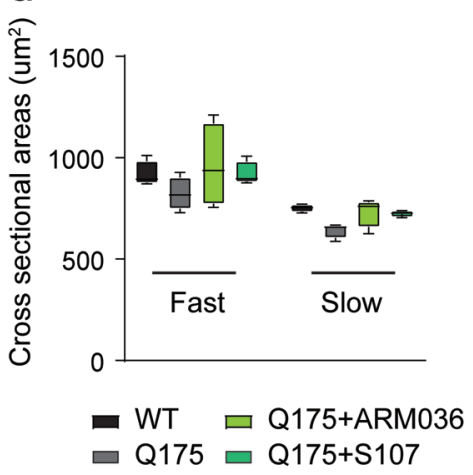

Q175 diaphragm

Po 0.17 To 6.89 ms Tc $74.7 \mathrm{~ms}$
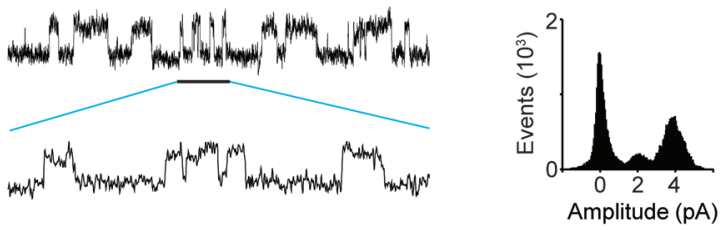

Q175 diaphragm + ARM036

Po 0.01 To 2.97 ms Tc $189.8 \mathrm{~ms}$
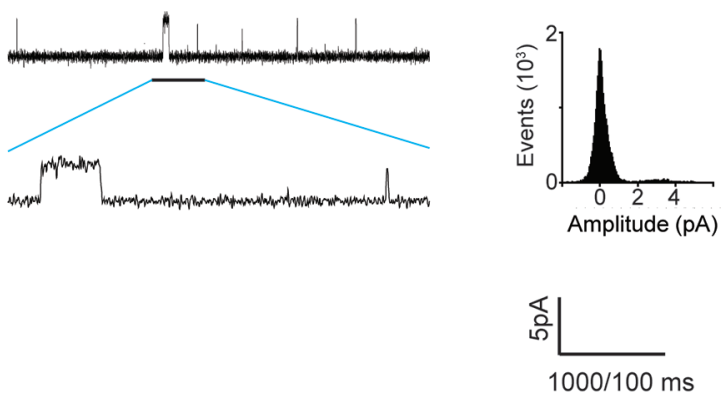

H

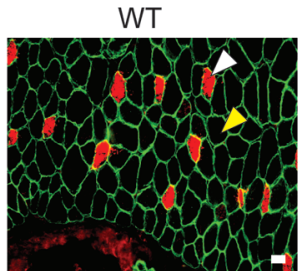

Q175+ARM036

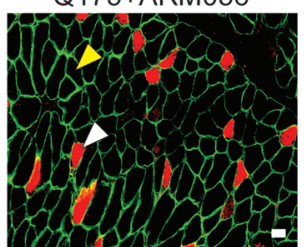

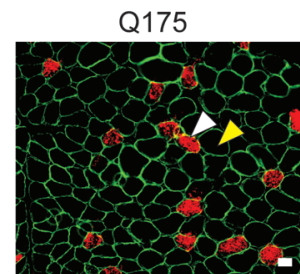

Q175+S107

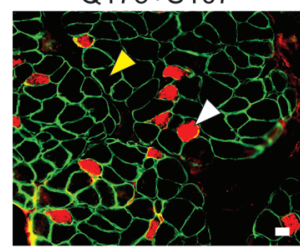


Figure 8. Diaphragmatic RyR1 remodeling in HD. (A and B) Representative SDS-PAGE analysis and quantification of RyR1 immunoprecipitated from diaphragm samples (band intensities were normalized to total RyR1) of WT $(n=6)$, Q175 $(n=6)$, Q175+ARM036 $(n=6)$, and Q175+S107 mice $(n=6)$. (C) Single-channel traces of RyR1 incorporated in planar lipid bilayers with $150 \mathrm{nM} \mathrm{Ca}{ }^{2+}$ in the cis chamber, corresponding to representative experiments performed with diaphragm samples from Q175 mice. (D-F) Increased RyR1 $P_{0}$ and $T_{0}$ and decreased Tc in Q175 mouse diaphragms. $P_{0}$ was $0.007 \pm 0.001$ in WT $(n=3)$ and in Q175 increased to $0.13 \pm 0.017(n=3)$ and restored to $0.017 \pm 0.02$ in Q175+S107 $(n=3)$ and to $0.010 \pm 0.002$ in Q175+ARM036 $(n=$ 3). Data (mean \pm SD) analysis was performed by 1-way ANOVA. Bonferroni's posttest revealed ${ }^{*} P<0.05$ vs. WT, ${ }^{\#} P<0.05$ vs. Q175. (G) Representative immunostaining of fast and slow diaphragm muscle fibers of WT $(n=6)$, Q175 $(n=6)$, Q175+ARM036 $(n=6)$, and Q175+S107 mice $(n=6)$. Quantified data are represented as a box-and-whisker plot, with bonds from 25 th to 75 th percentile, median line, and whiskers ranging from minimum to maximum values. Antibodies against fast-type (yellow arrows) and slow-type (white arrows) myosin ATPase were used to perform immunostaining on cryosections of mouse diaphragms. Muscle membrane was counterstained with dystrophin antibodies (green color). (H) Quantification of cross-sectional area (in $\mu m^{2}$ ) was calculated using ImageJ software (NIH) in each condition. Scale bar: $50 \mu \mathrm{m}$.

Heart failure occurs in about $30 \%$ of patients with HD and is a leading cause of death (86). Moreover, many patients with HD report respiratory symptoms at late stages of the disease, when the impaired motor control of the swallowing and respiratory muscle increases the risk of aspiration pneumonia $(7,14,87)$. However, little is known about the pathophysiological mechanism underlying the cardiorespiratory dysfunction in HD.

We found significant remodeling of RyR2 in the cortex and the hippocampus, likely related to cognitive function impairment, and in brainstem areas involved in autonomic regulation of cardiac activity (88). RyR2 remodeling includes PKA hyperphosphorylation, oxidation, nitrosylation, and calstabin depletion. These changes represent the biochemical signature of leaky RyR2. ER Ca ${ }^{2+}$ leak may contribute to neurodegeneration (54) and could explain in part the dysregulation of the ANS resulting in decreased HRV, which has been observed in patients with HD and in murine HD models $(25,27,88)$. Signaling from the central autonomic network is mediated through the preganglionic sympathetic and parasympathetic neurons. The most widespread cranial parasympathetic output is carried through the vagus nerve, which originates from the DMNV and NA and regulates cardiac activity. The increase in HR in Q175 mice is consistent with reduced parasympathetic activity. Our observations are consistent with the reduced HRV in patients with HD (89), including presymptomatic HD mutation carriers and mildly disabled HD patients (90). Further evidence of sympathovagal dysfunction includes symptoms such as orthostatic dizziness and tachycardia (25).

It has been suggested that sympathovagal dysautonomia in favor of sympathetic drive could result in fatal cardiac arrhythmias and/or promote heart failure in HD (28). Indeed, augmented sympathetic outflow and decreased vagal activity are considered proarrhythmic (91).

A complex network of neurons present mainly in the ventrolateral medulla oblongata and dorsolateral pons forms the respiratory central pattern generator (92). We found an overall brainstem remodeling of RyR2 and decreased diaphragmatic force production measured ex vivo in Q175 mice. In addition respiratory function was impaired at 10 months of age, manifested as a decreased TV and MV response to hypercapnia, indicative of a central respiratory defect. Interestingly, in a murine model of Duchenne muscular dystrophy, $m d x$ mice, there is normal breathing at rest despite diaphragmatic weakness (93, 94). Our results are in accordance with previous data obtained in patients with HD, where respiratory pressure, forced vital capacity, peak expiratory flow, and maximum voluntary ventilation were reduced compared with controls (13). HD symptoms have been suggested to be due to neurological dysfunction or secondary to a systemic illness due to mutated huntingtin protein expressed in the peripheral tissues $(2,95,96)$. To our knowledge, this is the first study evaluating the effects of huntingtin mutation in the brain, the heart, the diaphragm, and central versus peripheral pathophysiological mechanisms involved in HD. Because only S107 but not ARM036 prevented cardiorespiratory dysfunction, we conclude that leaky RyRs in the brainstem play an important role in HD pathology.

\section{Methods}

Animals. For the animal model, 10-month-old Q175-Z heterozygous (human mutant Htt [mHtt] allele with the expanded CAG repeat approximately 179 repeats within the native mouse huntingtin gene) mice and age-matched WT littermates and R6/2 (contains N-terminally truncated mHtt with CAG repeat expansion [ 125 repeats] within the HTT gene exon 1) were purchased from The Jackson Laboratory. Both male and female mice were used in this study. All in vivo animal experiments were performed by investigators blinded to genotype and treatment groups. 
S107 and ARM036 treatment. The Rycal S107 (BBB permeant) was administered in drinking water at $75 \mathrm{mg} / \mathrm{kg} / \mathrm{d}$ for 1 month as previously described (54). In order to differentiate between CNS effects versus peripheral muscle effects, the Rycal ARM036 (BBB nonpermeant) was administered in drinking water at 20 $\mathrm{mg} / \mathrm{kg} / \mathrm{d}$. Standard food was provided ad libitum throughout the experiments. Mouse weights and brain drug levels were as described in Supplemental Figure 3, B and C.

Immunoprecipitation. RyR1 and RyR2 were immunoprecipitated from the diaphragm, heart, and brain using an anti-RyR1 or anti-RyR2 antibody $(2 \mu \mathrm{g})$ in $0.5 \mathrm{~mL}$ of a modified RIPA buffer (50 mM Tris- $\mathrm{HCl}$ at $\mathrm{pH} 7.2,0.9 \% \mathrm{NaCl}, 5.0 \mathrm{mM} \mathrm{NaF}, 1.0 \mathrm{mM} \mathrm{Na}_{3} \mathrm{VO}_{4}, 1 \%$ Triton $\mathrm{X}-100$, and protease inhibitors) overnight at $4^{\circ} \mathrm{C}$. The RyR1-specific antibody was RyR1-1327, an affinity-purified rabbit polyclonal antibody raised against a keyhole limpet hemocyanin-conjugated peptide with the amino acid sequence CAEPDTDYENLRRS, corresponding to residues 1327-1339 of mouse skeletal RyR1, with an additional cysteine residue added to the amino terminus, and affinity purified with the unconjugated peptide (78). The RyR2-specific antibody was an affinity-purified polyclonal rabbit antibody using the peptide CKPEFNNHKDYAQEK corresponding to amino acids $1367-1380$ of mouse RyR2 with a cysteine residue added to the amino terminus (60). The immune complexes were incubated with protein A-Sepharose beads (MilliporeSigma) at $4^{\circ} \mathrm{C}$ for 1 hour, and the beads were washed 3 times with RIPA buffer. The immunoprecipitates were size fractionated on SDS-PAGE gels (4\%-20\% for RyR1/RyR2 and calstabin) and transferred onto nitrocellulose membranes for 2 hours at $200 \mathrm{~mA}$. Immunoblots were developed using the following primary antibodies: anti-RyR1/2 (Affinity BioReagents, 1:2000, ab55999), anti-phospho-RyR-Ser(P)-2808 (Affinity BioReagents, 1:5000, ab59225), anti-calstabin (FKBP12 C-19, SC-133067, 1:1000, Santa Cruz Biotechnology, Inc.), and anti-CysNO (Y061263, 1:1000, MilliporeSigma). To determine channel oxidation, the carbonyl groups in the protein side chains were derivatized to DNP by reaction with 2,4-dinitrophenylhydrazine. The DNP signal associated with RyR was determined using a specific anti-DNP antibody according to the manufacturer's instructions (MilliporeSigma S7150). All immunoblots were developed using an Odyssey system (LI-COR Biosciences) with infrared-labeled anti-mouse and anti-rabbit IgG (1:10,000, 926-32211) secondary antibodies.

$S R$ vesicle preparation. Brains, hearts, and diaphragms were homogenized on ice in $300 \mathrm{mM}$ sucrose, with $20 \mathrm{mM}$ 1,4-piperazinediethanesulfonic acid (PIPES, MilliporeSigma) ( $\mathrm{pH}$ 7.0) in the presence of protease inhibitors (Roche), and centrifuged at $5900 \mathrm{~g}$ for 20 minutes at $4^{\circ} \mathrm{C}$. The supernatant was ultracentrifuged at $100,000 \mathrm{~g}$ for 1 hour at $4^{\circ} \mathrm{C}$. The final pellet containing microsomal fractions enriched in SR vesicles was resuspended and aliquoted in $300 \mathrm{mM}$ sucrose, with $5 \mathrm{mM}$ PIPES (pH 7.0) containing protease inhibitors. Samples were frozen in liquid nitrogen and stored at $-80^{\circ} \mathrm{C}$.

Single-channel data using planar lipid bilayers. Planar lipid bilayers were formed using a 3:1 mixture of phosphatidylethanolamine and phosphatidylcholine (Avanti Polar Lipids) suspended (30 mg/ $\mathrm{mL}$ ) in decane by painting the lipid/decane solution across a $200 \mu \mathrm{m}$ aperture in a polysulfonate cup (Warner Instruments) separating 2 chambers. The trans chamber $(1 \mathrm{~mL})$ representing the intra-ER/ SR (luminal) compartment was connected to the headstage input of a bilayer voltage clamp amplifier (BC-525D, Warner Instruments), and the cis chamber $(1 \mathrm{~mL})$, representing the cytoplasmic compartment, was held at virtual ground. Solutions in both chambers were as follows: $1 \mathrm{mM}$ EGTA, 250/125 $\mathrm{mM}$ HEPES/Tris, $50 \mathrm{mM} \mathrm{KCl}$, and $0.64 \mathrm{mM} \mathrm{CaCl}_{2}, \mathrm{pH} 7.35$ as cis solution and $53 \mathrm{mM} \mathrm{Ca}(\mathrm{OH})_{2}, 50$ $\mathrm{mM} \mathrm{KCl}, 2$ and $50 \mathrm{mM}$ HEPES, pH 7.35 as trans solution. The concentration of free $\mathrm{Ca}^{2+}$ in the cis chamber was calculated using the WinMaxC program (version 2.50). SR vesicles were added to the cis side, and fusion with the lipid bilayer was induced by making the cis side hyperosmotic by the addition of $400-500 \mathrm{mM} \mathrm{KCl}$. After the appearance of potassium and chloride channels, the cis compartment was perfused with the cis solution. Single-channel currents were recorded at $0 \mathrm{mV}$ by using a Bilayer Clamp BC-535 amplifier (Warner Instruments), filtered at $1 \mathrm{kHz}$, and digitized at $4 \mathrm{kHz}$. All experiments were performed at room temperature. Data acquisition was performed using Digidata 1440A and Axoscope 10.2 software; recordings were analyzed using Clampfit 10.2 (Molecular Devices, Thermo Fisher Scientific). $\mathrm{P}_{\mathrm{O}}$ was identified by $50 \%$ threshold analyses using a minimum of 2 minutes of continuous record. At the conclusion of each experiment, ryanodine $(5 \mu \mathrm{M})$ was added to the cis chamber to confirm channels as RyR.

Brain immunohistochemistry. Mice were perfused transcardially with $30 \mathrm{~mL}$ phosphate-buffered saline followed by $30 \mathrm{~mL}$ of $4 \%$ formaldehyde. Brainstems were removed and postfixed for 12 hours in $4 \%$ formaldehyde at $4{ }^{\circ} \mathrm{C}$. All brainstems were cut coronally (50 $\mu \mathrm{m}$ sections) using a vibratome. Fluorescence immunohistochemistry was performed as previously described $(97,98)$. Sections were immunostained for 
detection of TH, ChAT, and either RyR2 or P*RyR2. The primary antibodies used were mouse anti-TH (1:500, Merck, MAB318), goat anti-ChAT (1:200, Merck, AB144P), rabbit anti-RyR2 (60) (1:400), and rabbit anti-P*RyR2 (1:400, ab59225, Affinity BioReagents). The secondary antibodies used were Alexa Fluor 647 donkey anti-mouse (1:500, Invitrogen, Thermo Fisher Scientific, A31571), Alexa Fluor 555 donkey anti-rabbit (1:500, Invitrogen, Thermo Fisher Scientific, A31572), and Alexa Fluor 488 donkey anti-goat (1:500, Invitrogen, Thermo Fisher Scientific, A11055). The presence of RyR2 or P*RyR2 immunoreactivity in presympathetic adrenergic $\mathrm{C} 1$ neurons of the rostral ventrolateral medulla oblongata (TH immunoreactive) and cardiac parasympathetic preganglionic neurons of the NA (ChAT immunoreactive) was examined using a ZEISS Axioplan 2 microscope with an Apotome module. Brainstem sections were sampled every $100 \mu \mathrm{m}$, and bilateral images ( $\times 20$ objective) of typically $2-3$ sections per animal were acquired using 9 tiles of $Z$-stacks composed of 8 optical sections ( $8 \mu \mathrm{m}$ focal spacing). For Figure 3A, a maximum intensity projection of optical sections was performed. Semiquantitative analyses of colocalization between RyR 2 or $\mathrm{P} *$ RyR2 immunoreactivity and TH or ChAT immunoreactivity were performed in 4 WT and 4 Q175 mice, using the following code: $-=0$ colocalized neurons; $+=1 \%-25 \%$ colocalized neurons; $++=26 \%-50 \%$ colocalized neurons; $+++=51 \%-75 \%$ colocalized neurons; and $++++=76 \%-100 \%$ colocalized neurons.

Measurement of diaphragm fibers' cross-sectional areas. Frozen diaphragm muscle strips from the left middle segment of costal diaphragms were embedded in OCT for cryosections. Cryosections $(10 \mu \mathrm{m})$ were taken precisely perpendicular to the fibers by a previously described technique (99), fixed with cold isopentane for 2 minutes, and immunostained with murine anti- $\alpha$-myosin heavy chain (MilliporeSigma, 1:2000, M7421). Muscle membrane was counterstained with rabbit anti-dystrophin antibodies (Abcam, 1:1000, ab230379) using standard procedures. Alexa Fluor 555 anti-mouse and Alexa Fluor 488 anti-rabbit secondary antibody (Thermo Fisher Scientific A11094 and A32727) were then applied. Slides were mounted in ProLong Gold antifading reagent (Invitrogen, Thermo Fisher Scientific) and imaged by confocal fluorescence microscopy. The cross-sectional area (CSA) was measured using Fiji (http://fiji.sc/wiki/index.php/Fiji) software, an enhanced version of ImageJ (NIH). Two regions from each section (total $~ 150$ fibers/sample) were processed.

Contractile function in murine muscle samples. Mice were euthanized by exsanguination and the entire diaphragm EDL or soleus was surgically excised. Isometric contractile properties were assessed as described elsewhere (100). The excised diaphragm strip, EDL, and soleus were mounted into jacketed tissue bath chambers filled with equilibrated and oxygenated Krebs solution. The muscles were supramaximally stimulated using square wave pulses (Model S48; Grass Instruments). The force-frequency relationship was determined by sequentially stimulating the muscles for $600 \mathrm{~ms}$ at 10, 20, 30, $50,60,80,100$, and $120 \mathrm{~Hz}$ with 1 minute between each stimulation train (53). After measurement of contractile properties, muscles were measured at the length at which the muscle produced maximal isometric tension, dried, and weighed. For comparative purposes, muscle force production was normalized for total muscle strip CSA and expressed in $\mathrm{N} / \mathrm{cm}^{2}$. The total muscle strip CSA was determined by dividing muscle weight by its length and tissue density $\left(1.056 \mathrm{~g} / \mathrm{cm}^{3}\right)$.

Behavioral studies. EPM and MWM tests were performed as previously described (54, 58). Novel object recognition test was performed as previously described (101). Briefly, the EPM test was performed using the EPM apparatus, which consisted of 4 arms ( 2 open without walls and 2 enclosed by $15 \mathrm{~cm}$ high walls), $68 \mathrm{~cm}$ long and $5 \mathrm{~cm}$ wide, was elevated $55 \mathrm{~cm}$ off the floor. A video tracking system (Noldus Information Technology Inc.) with computer interface and video camera were used to automatically collect behavioral data. Each mouse was placed at the junction of the 4 arms of the maze; numbers of entries and the time spent in each arm were recorded by the examiner and the video tracking system simultaneously for 5 minutes. Deodar wiper was used between each tested mouse. The ratio of total time spent in, and the numbers of entry to the open arm versus closed arm, of each mouse were analyzed.

Spatial learning and memory function were evaluated using MWM (102) task, which consisted of a circular pool (122 cm in diameter, $76 \mathrm{~cm}$ in depth) (San Diego Instruments) in which mice were trained to escape from water by swimming to a $10 \mathrm{~cm}$ diameter hidden platform $(1.0$ to $1.5 \mathrm{~cm}$ underneath water surface). Water temperature was maintained at $23^{\circ} \mathrm{C} \pm 1^{\circ} \mathrm{C}$, and the water was rendered opaque by the addition of white nontoxic paint (Discount School Supply). The pool was divided into 4 quadrants by a computerized tracking/image analyzing system (Noldus Information Technology Inc.). The hidden platform was placed in the middle of the northwest (NW) quadrant and remained in the same position during the experiment. The spatial acquisition phase consisted of 15 training trials: 5 training days and 3 trials per day with an intertrial interval of 40-60 minutes. Mice were released from 
different quadrants between trials with their heads facing the pool wall of 1 of the 4 compass locations (SW, SE, and NE) and allowed to swim and search for the platform for 60 seconds. The latency to reach the hidden platform, travel path, and swimming velocity were recorded by the examiner and the video tracking system simultaneously. On day 6, memory retention was evaluated by a probe trial 24 hours after the last training session in the absence of the escape platform. Mice were allowed to swim freely for 60 seconds. The location where the hidden platform was previously located was defined as the target. The northwest quadrant where the platform was previously hidden was defined as the target quadrant. The number of target crossings and the proportion of swimming time spent in the target quadrant were recorded and analyzed.

The tail suspension test was used to assess depression-related behavior, as described previously (102). Briefly, on the top of a box, a suspension bar was used to suspend the tail of the mouse. Each mouse was suspended separately in the middle of the box, using black adhesive tape applied to the end of the tail (with 2-3 mm remaining outside the tape), and the free end of the tape was attached to the middle of the suspension bar. The approximate distance between the mouse's nose and the apparatus floor was 10-15 cm. Every session lasted for 6 minutes and was recorded using a video camera placed on a tripod in front of the box. The time that each mouse spent immobile was measured. Lack of escape-related behavior is considered immobility, which is indicative of depression-like behavior.

Whole-body plethysmography measurement. Respiratory function was measured in conscious, unrestrained mice using whole-body plethysmography and analyzed using iox2 software (EMKA Technologies) as previously described $(94,103)$. Mice were randomly placed into individual chambers. After 1-hour stabilization, each animal was visually monitored, and ventilatory parameters were recorded during an inactive phase for 5 minutes under 2 experimental conditions: first, room air breathing, then under hypercapnic conditions using $8 \% \mathrm{CO}_{2}$-enriched airflow to activate respiration. Air volume changes corresponding to spontaneous breathing were obtained using a pressure transducer (EMKA Technologies). This pressure signal was used to calculate ventilatory parameters: TV, respiratory rate, $\mathrm{MV}$, inspiratory time, and expiratory time.

Running wheel. Mice were subjected to voluntary aerobic exercise with free access to a running wheel (diameter $11.5 \mathrm{~cm}$ ), which was connected to a digital counter for 24 hours (light/dark cycle), as previously described (104).

ECG recording in conscious animals. Mice were implanted with radio telemetry transmitters (Data Sciences International) as described in detail elsewhere (105). Briefly, the transmitter (PhysioTel, ETA-F10 transmitter) was inserted in mice subcutaneously along the back under general anesthesia (20\% inhaled isoflurane $/ \mathrm{O}_{2}$, Aerrane) coupled with local anesthetic (lidocaine 0.5\%), and 2 ECG electrodes were placed hypodermically in the region of the right shoulder (negative pole) and toward the lower left chest (positive pole) to approximate lead II of the Einthoven surface ECG. During the procedure, respiratory and cardiac rhythm, adequacy of anesthetic depth, muscle relaxation, body temperature, and analgesia were monitored to avoid anesthesia-related complications. Postoperating pain was considered during a 1-week postimplantation period and buprenorphine $(0.3 \mathrm{mg} / \mathrm{kg}$ s.c.) was given. A minimum period of 2 weeks was allowed for recovery from the surgery. Animals were housed in individual stainless steel cages for telemetry recordings. Environmental parameters were recorded continuously and maintained within a fixed range: room temperature at $15^{\circ} \mathrm{C}-21^{\circ} \mathrm{C}$ and $45 \%-65 \%$ relative humidity. The artificial day/ night cycle was 12-hour light/12-hour dark with light on at 0700 hours. Drinking water was provided ad libitum. Solid diet (300 g) was given daily in the morning. ECG waveforms were continuously recorded at a sampling rate of $2000 \mathrm{~Hz}$ using a signal transmitter-receiver (RPC-1) connected to a data acquisition system (Ponemah system, Data Sciences International). Continuous digital recordings were analyzed offline after being digitally filtered between 0.1 and $1000 \mathrm{~Hz}$. ECGs during nocturnal and diurnal periods (12-hour light/12-hour dark cycle) were analyzed with Ponemah software using template automatic detection, secondly validated by an operator. The mean RR interval and QT durations were calculated. The QT interval was defined as the time between the first deviation from an isoelectric PR interval until the return of the ventricular repolarization to the isoelectric TP baseline from lead II ECGs.

Presence of ectopic beats was scanned by hand. Then, HRV was evaluated by power spectral analysis $\left(\mathrm{ms}^{2}\right)$ using the fast Fourier transformation (segment length of 2048 beats, linear interpolation with resampling to a $20 \mathrm{~Hz}$ interbeat time series and Hamming windowing). The cutoff frequency ranges for the LFr powers (HFr: 1.5-5 Hz) were chosen according to those used in the literature (106). 
Autonomic blockade. Complete suppression of autonomic control was achieved with ganglioplegic compound hexamethonium (20 mg/kg, i.p.).

Further information can be found in Supplemental Methods.

Statistics. Group data are presented as mean \pm SD unless otherwise indicated. Statistical comparisons between 2 groups were tested using an unpaired 2-tailed $t$ test. ANOVA tests ( 1 and 2 way) with a Bonferroni's post hoc adjustment were used for multiple comparisons. Values of $P<0.05$ were considered statistically significant. All statistical analyses were performed with GraphPad Prism 8.0.

Study approval. For human samples, deidentified human hippocampus and cortex samples were obtained from the New York Brain Bank at Columbia University. The age and sex of these samples were 64, female; 58, female; 54, female; 61, female; 66, female; 56, male; 58, male; and 63, male. Age- and sexmatched controls exhibited absence of neurological disorders and plaques, and previous experiments using these specific control samples had shown a lack of remodeling and leak in RyR2 (54). Information on the patients with HD is listed in Supplemental Table 1.

For the animal model, all mice were maintained and studied according to protocols approved by the Institutional Animal Care and Use Committee of Columbia University (reference AC-AAAV5455) and Directive 2010/63/EU of the European parliament and the Council of September 22, 2010, for the protection of animals used for scientific purposes and ethics committee for animals experiments, Languedoc Roussillon, C2EA-36 (agreement B34-172-38; project APAFIS 13528-2018). Q111 frozen brain tissues were obtained from the CHDI Foundation (New York, New York, USA) for only RyR2 analysis. We chose 10-month-old Q175 mice when the mice exhibited remarkable behavioral deficits accompanied by marked brain atrophy and brain metabolite changes and started developing respiratory and autonomic dysfunction (107).

\section{Author contributions}

$\mathrm{HD}, \mathrm{AL}$, and ARM designed experiments, analyzed data, and edited or wrote the paper. XL, QY, SR, MY, LS, PA, JT, PS, SM, CM, and JB designed experiments and analyzed data.

\section{Acknowledgments}

This work was supported by grants from the NIH to ARM (T32HL120826, R01HL145473, R01DK118240, R01HL142903, R01HL061503, R01HL140934, R01AR070194) and by the CHDI Foundation.

Address correspondence to: Andrew R. Marks, 1150 St. Nicholas Avenue, Room 520, New York, New York 10032, USA. Phone: 212.851.5340; Email: arm42@cumc.columbia.edu. Or to: Alain Lacampagne, UMR CNRS 9214, INSERM U1046, Physiologie et Medecine experimental du Coeur et des muscles, CHU Arnaud de Villeneuve 34295 Montpellier Cedex 05, France. Phone: 33.67.415.228; Email: alain. lacampagne@inserm.fr.

1. Harper PS. Huntington disease and the abuse of genetics. Am J Hum Genet. 1992;50(3):460-464.

2. Walker FO. Huntington's disease. Semin Neurol. 2007;27(2):143-150.

3. Singhrao SK, et al. Huntingtin protein colocalizes with lesions of neurodegenerative diseases: an investigation in Huntington's, Alzheimer's, and Pick's diseases. Exp Neurol. 1998;150(2):213-222.

4. van der Burg JM, Björkqvist M, Brundin P. Beyond the brain: widespread pathology in Huntington's disease. Lancet Neurol. 2009;8(8):765-774.

5. Roos RA. Huntington's disease: a clinical review. Orphanet J Rare Dis. 2010;5:40.

6. Heemskerk AW, Roos RA. Aspiration pneumonia and death in Huntington's disease. PLoS Curr. 2012;4:RRN1293.

7. Heemskerk AW, Roos RA. Dysphagia in Huntington's disease: a review. Dysphagia. 2011;26(1):62-66.

8. Jones $\mathrm{K}$, et al. Interventions for dysphagia in long-term, progressive muscle disease. Cochrane Database Syst Rev. 2016;2:CD004303.

9. Jones U, Enright S, Busse M. Management of respiratory problems in people with neurodegenerative conditions(1): a narrative review. Physiotherapy. 2012;98(1):1-12.

10. Mehanna R, Jankovic J. Respiratory problems in neurologic movement disorders. Parkinsonism Relat Disord. 2010;16(10):628-638.

11. Zinzi P, et al. Effects of an intensive rehabilitation programme on patients with Huntington's disease: a pilot study. Clin Rehabil. 2007;21(7):603-613

12. Busse ME, Khalil H, Quinn L, Rosser AE. Physical therapy intervention for people with Huntington disease. Phys Ther. 2008;88(7):820-831.

13. Reyes A, Cruickshank T, Ziman M, Nosaka K. Pulmonary function in patients with Huntington's disease. BMC Pulm Med. 2014;14:89. 
14. Gil JM, Rego AC. Mechanisms of neurodegeneration in Huntington's disease. Eur J Neurosci. 2008;27(11):2803-2820.

15. Abildtrup M, Shattock M. Cardiac dysautonomia in Huntington's disease. J Huntingtons Dis. 2013;2(3):251-261.

16. Chiu E, Alexander L. Causes of death in Huntington's disease. Med J Aust. 1982;1(4):153.

17. Conneally PM. Huntington disease: genetics and epidemiology. Am J Hum Genet. 1984;36(3):506-526.

18. Haines JL, Conneally PM. Causes of death in Huntington disease as reported on death certificates. Genet Epidemiol. 1986;3(6):417-423.

19. Lanska DJ, Lavine L, Lanska MJ, Schoenberg BS. Huntington's disease mortality in the United States. Neurology. 1988;38(5):769-772.

20. Sørensen SA, Fenger K. Causes of death in patients with Huntington's disease and in unaffected first degree relatives. $J M e d$ Genet. 1992;29(12):911-914.

21. Heemskerk AW, Roos RA. Aspiration pneumonia and death in Huntington's disease. PLoS Curr. 2012;4:RRN1293.

22. Schönberger SJ, Jezdic D, Faull RL, Cooper GJ. Proteomic analysis of the human brain in Huntington's Disease indicates pathogenesis by molecular processes linked to other neurodegenerative diseases and to type-2 diabetes. J Huntingtons Dis 2013;2(1):89-99.

23. Stephen CD, Hung J, Schifitto G, Hersch SM, Rosas HD. Electrocardiogram abnormalities suggest aberrant cardiac conduction in Huntington's disease. Mov Disord Clin Pract. 2018;5(3):306-311.

24. Andrich J, et al. Autonomic nervous system function in Huntington's disease. J Neurol Neurosurg Psychiatry. 2002;72(6):726-731.

25. Bär KJ, et al. Cardiovagal modulation upon postural change is altered in Huntington's disease. Eur J Neurol. 2008;15(8):869-871.

26. Den Heijer JC, et al. Autonomic nervous function in Huntington's disease. Arch Neurol. 1988;45(3):309-312.

27. Sharma KR, Romano JG, Ayyar DR, Rotta FT, Facca A, Sanchez-Ramos J. Sympathetic skin response and heart rate variability in patients with Huntington disease. Arch Neurol. 1999;56(10):1248-1252.

28. Kobal J, et al. Autonomic dysfunction in presymptomatic and early symptomatic Huntington's disease. Acta Neurol Scand. 2010;121(6):392-399.

29. Virlogeux A, et al. Reconstituting corticostriatal network on-a-chip reveals the contribution of the presynaptic compartment to Huntington's disease. Cell Rep. 2018;22(1):110-122.

30. Tyebji S, Hannan AJ. Synaptopathic mechanisms of neurodegeneration and dementia: insights from Huntington's disease. Prog Neurobiol. 2017;153:18-45.

31. Park H. Cortical axonal secretion of BDNF in the striatum is disrupted in the mutant-huntingtin knock-in mouse model of Huntington's disease. Exp Neurobiol. 2018;27(3):217-225.

32. Yu C, Li CH, Chen S, Yoo H, Qin X, Park H. Decreased BDNF release in cortical neurons of a knock-in mouse model of Huntington's disease. Sci Rep. 2018;8(1):16976.

33. Carmo C, Naia L, Lopes C, Rego AC. Mitochondrial dysfunction in Huntington's disease. Adv Exp Med Biol. 2018;1049:59-83

34. Franco-Iborra S, Vila M, Perier C. Mitochondrial quality control in neurodegenerative diseases: focus on Parkinson's disease and Huntington's disease. Front Neurosci. 2018;12:342.

35. Bezprozvanny I. Calcium signaling and neurodegenerative diseases. Trends Mol Med. 2009;15(3):89-100

36. Miller BR, Bezprozvanny I. Corticostriatal circuit dysfunction in Huntington's disease: intersection of glutamate, dopamine and calcium. Future Neurol. 2010;5(5):735-756

37. Raymond LA. Striatal synaptic dysfunction and altered calcium regulation in Huntington disease. Biochem Biophys Res Commun. 2017;483(4):1051-1062.

38. Tang TS, Tu H, Wang Z, Bezprozvanny I. Modulation of type 1 inositol (1,4,5)-trisphosphate receptor function by protein kinase a and protein phosphatase 1alpha. J Neurosci. 2003;23(2):403-415.

39. Wu J, et al. Enhanced store-operated calcium entry leads to striatal synaptic loss in a Huntington's disease mouse model. J Neurosci. 2016;36(1):125-141.

40. Suzuki M, Nagai Y, Wada K, Koike T. Calcium leak through ryanodine receptor is involved in neuronal death induced by mutant huntingtin. Biochem Biophys Res Commun. 2012;429(1-2):18-23.

41. de Pascual R, Álvarez-Ortego N, de Los Ríos C, Jacob-Mazariego G, García AG. Tetrabenazine facilitates exocytosis by enhancing calcium-induced calcium release through ryanodine receptors. J Pharmacol Exp Ther. 2019;371(1):219-230.

42. Nekrasov ED, Kiselev SL. Mitochondrial distribution violation and nuclear indentations in neurons differentiated from iPSCs of Huntington's disease patients. J Stem Cells Regen Med. 2018;14(2):80-85.

43. Rosemblit N, Moschella MC, Ondriašová E, Gutstein DE, Ondriaš K, Marks AR. Intracellular calcium release channel expression during embryogenesis. Dev Biol. 1999;206(2):163-177.

44. Santulli G, Lewis D, des Georges A, Marks AR, Frank J. Ryanodine receptor structure and function in health and disease. Subcell Biochem. 2018;87:329-352.

45. Brillantes AB, et al. Stabilization of calcium release channel (ryanodine receptor) function by FK506-binding protein. Cell. 1994;77(4):513-523

46. Marks AR, et al. Molecular cloning and characterization of the ryanodine receptor/junctional channel complex cDNA from skeletal muscle sarcoplasmic reticulum. Proc Natl Acad Sci U S A. 1989;86(22):8683-8687.

47. Marx SO, et al. PKA phosphorylation dissociates FKBP12.6 from the calcium release channel (ryanodine receptor): defective regulation in failing hearts. Cell. 2000;101(4):365-376.

48. Sorrentino V. Ryanodine receptor type 3: why another ryanodine receptor isoform? Front Biosci. 2003;8:d176-d182.

49. Maki T, et al. Regulation of calcium channel expression in neonatal myocytes by catecholamines. J Clin Invest. 1996;97(3):656-663.

50. Marks AR, Marx SO, Reiken S. Regulation of ryanodine receptors via macromolecular complexes: a novel role for leucine/ isoleucine zippers. Trends Cardiovasc Med. 2002;12(4):166-170.

51. Callaway $\mathrm{C}$, et al. Localization of the high and low affinity $[3 \mathrm{H}]$ ryanodine binding sites on the skeletal muscle Ca2+ release channel. J Biol Chem. 1994;269(22):15876-15884.

52. Xie W, et al. Mitochondrial oxidative stress promotes atrial fibrillation. Sci Rep. 2015;5:11427.

53. Umanskaya A, Santulli G, Xie W, Andersson DC, Reiken SR, Marks AR. Genetically enhancing mitochondrial antioxidant activity improves muscle function in aging. Proc Natl Acad Sci U S A. 2014;111(42):15250-15255. 
54. Liu X, et al. Role of leaky neuronal ryanodine receptors in stress-induced cognitive dysfunction. Cell. 2012;150(5):1055-1067.

55. Lehnart SE, et al. Phosphodiesterase 4D deficiency in the ryanodine-receptor complex promotes heart failure and arrhythmias. Cell. 2005;123(1):25-35.

56. Shan J, et al. Role of chronic ryanodine receptor phosphorylation in heart failure and $\beta$-adrenergic receptor blockade in mice. J Clin Invest. 2010;120(12):4375-4387.

57. Kushnir A, Shan J, Betzenhauser MJ, Reiken S, Marks AR. Role of CaMKIIdelta phosphorylation of the cardiac ryanodine receptor in the force frequency relationship and heart failure. Proc Natl Acad Sci U S A. 2010;107(22):10274-10279.

58. Lacampagne A, et al. Post-translational remodeling of ryanodine receptor induces calcium leak leading to Alzheimer's disease-like pathologies and cognitive deficits. Acta Neuropathol. 2017;134(5):749-767.

59. Ward CW, Reiken S, Marks AR, Marty I, Vassort G, Lacampagne A. Defects in ryanodine receptor calcium release in skeletal muscle from post-myocardial infarct rats. FASEB J. 2003;17(11):1517-1519.

60. Bussiere R, et al. Amyloid $\beta$ production is regulated by $\beta 2$-adrenergic signaling-mediated post-translational modifications of the ryanodine receptor. J Biol Chem. 2017;292(24):10153-10168.

61. Bellinger AM, et al. Hypernitrosylated ryanodine receptor calcium release channels are leaky in dystrophic muscle. Nat Med. 2009;15(3):325-330.

62. Bellinger AM, et al. Remodeling of ryanodine receptor complex causes "leaky" channels: a molecular mechanism for decreased exercise capacity. Proc Natl Acad Sci U S A. 2008;105(6):2198-2202.

63. Bellinger AM, Mongillo M, Marks AR. Stressed out: the skeletal muscle ryanodine receptor as a target of stress. J Clin Invest. 2008;118(2):445-453.

64. Andersson DC, et al. Ryanodine receptor oxidation causes intracellular calcium leak and muscle weakness in aging. Cell Metab. 2011;14(2):196-207.

65. Andersson DC, et al. Leaky ryanodine receptors in $\beta$-sarcoglycan deficient mice: a potential common defect in muscular dystrophy. Skelet Muscle. 2012;2(1):9.

66. Chen X, Wu J, Lvovskaya S, Herndon E, Supnet C, Bezprozvanny I. Dantrolene is neuroprotective in Huntington's disease transgenic mouse model. Mol Neurodegener. 2011;6:81.

67. Giannini G, Conti A, Mammarella S, Scrobogna M, Sorrentino V. The ryanodine receptor/calcium channel genes are widely and differentially expressed in murine brain and peripheral tissues. J Cell Biol. 1995;128(5):893-904.

68. Lawrence AD, Sahakian BJ, Robbins TW. Cognitive functions and corticostriatal circuits: insights from Huntington's disease. Trends Cogn Sci (Regul Ed). 1998;2(10):379-388.

69. Grahn JA, Parkinson JA, Owen AM. The role of the basal ganglia in learning and memory: neuropsychological studies. Behav Brain Res. 2009;199(1):53-60.

70. Shan J, et al. Calcium leak through ryanodine receptors leads to atrial fibrillation in 3 mouse models of catecholaminergic polymorphic ventricular tachycardia. Circ Res. 2012;111(6):708-717.

71. Santulli G, Marks AR. Essential roles of intracellular calcium release channels in muscle, brain, metabolism, and aging. Curr Mol Pharmacol. 2015;8(2):206-222.

72. Marx SO, et al. Phosphorylation-dependent regulation of ryanodine receptors: a novel role for leucine/isoleucine zippers. J Cell Biol. 2001;153(4):699-708.

73. Guyenet PG, Stornetta RL, Bochorishvili G, Depuy SD, Burke PG, Abbott SB. C1 neurons: the body's EMTs. Am J Physiol Regul Integr Comp Physiol. 2013;305(3):R187-R204.

74. Champeroux $\mathrm{P}$, et al. Short-term variability in QT interval and ventricular arrhythmias induced by dofetilide are dependent on high-frequency autonomic oscillations. Br J Pharmacol. 2015;172(11):2878-2891.

75. Thireau J, Zhang BL, Poisson D, Babuty D. Heart rate variability in mice: a theoretical and practical guide. Exp Physiol. 2008;93(1):83-94

76. Wehrens $\mathrm{XH}$, et al. Protection from cardiac arrhythmia through ryanodine receptor-stabilizing protein calstabin2. Science. 2004;304(5668):292-296.

77. Oleynikov VE, Galimskaya VA, Kupriyanova SN, Burko NV. Use of the Speckle tracking method for determining global parameters of heart contractility in healthy individuals. Methods X. 2018;5:125-135.

78. Matecki S, et al. Leaky ryanodine receptors contribute to diaphragmatic weakness during mechanical ventilation. Proc Natl Acad Sci U S A. 2016;113(32):9069-9074.

79. Harnick DJ, Jayaraman T, Ma Y, Mulieri P, Go LO, Marks AR. The human type 1 inositol 1,4,5-trisphosphate receptor from T lymphocytes. Structure, localization, and tyrosine phosphorylation. J Biol Chem. 1995;270(6):2833-2840.

80. Suzuki M, Nagai Y, Wada K, Koike T. Calcium leak through ryanodine receptor is involved in neuronal death induced by mutant huntingtin. Biochem Biophys Res Commun. 2012;429(1-2):18-23.

81. Hansson O, et al. Resistance to NMDA toxicity correlates with appearance of nuclear inclusions, behavioural deficits and changes in calcium homeostasis in mice transgenic for exon 1 of the huntington gene. Eur J Neurosci. 2001;14(9):1492-1504.

82. Okuda S, et al. CaMKII-mediated phosphorylation of RyR2 plays a crucial role in aberrant $\mathrm{Ca}^{2+}$ release as an arrhythmogenic substrate in cardiac troponin T-related familial hypertrophic cardiomyopathy. Biochem Biophys Res Commun. 2018;496(4):1250-1256.

83. Joviano-Santos JV, et al. Increased oxidative stress and CaMKII activity contribute to electro-mechanical defects in cardiomyocytes from a murine model of Huntington's disease. FEBS J. 2019;286(1):110-123.

84. Deckel AW, Elder R, Fuhrer G. Biphasic developmental changes in Ca2+/calmodulin-dependent proteins in R6/2 Huntington's disease mice. Neuroreport. 2002;13(5):707-711.

85. Ferreira IL, Carmo C, Naia L, I Mota S, Cristina Rego A. Assessing mitochondrial function in in vitro and ex vivo models of Huntington's disease. Methods Mol Biol. 2018;1780:415-442.

86. Lanska DJ, Lanska MJ, Lavine L, Schoenberg BS. Conditions associated with Huntington's disease at death. A case-control study. Arch Neurol. 1988;45(8):878-880.

87. Niedringhaus M, Jackson PG, Evans SR, Verbalis JG, Gillis RA, Sahibzada N. Dorsal motor nucleus of the vagus: a site for evoking simultaneous changes in crural diaphragm activity, lower esophageal sphincter pressure, and fundus tone. Am J Physiol Regul Integr Comp Physiol. 2008;294(1):R121-R131. 
88. Benarroch EE. The central autonomic network: functional organization, dysfunction, and perspective. Mayo Clin Proc. 1993;68(10):988-1001

89. Aziz NA, Anguelova GV, Marinus J, van Dijk JG, Roos RA. Autonomic symptoms in patients and pre-manifest mutation carriers of Huntington's disease. Eur J Neurol. 2010;17(8):1068-1074.

90. Kobal J, Meglic B, Mesec A, Peterlin B. Early sympathetic hyperactivity in Huntington's disease. Eur J Neurol. 2004;11(12):842-848.

91. Ng GA, Brack KE, Patel VH, Coote JH. Autonomic modulation of electrical restitution, alternans and ventricular fibrillation initiation in the isolated heart. Cardiovasc Res. 2007;73(4):750-760.

92. Smith JC, Abdala AP, Borgmann A, Rybak IA, Paton JF. Brainstem respiratory networks: building blocks and microcircuits. Trends Neurosci. 2013;36(3):152-162.

93. Gayraud J, et al. Ventilation during air breathing and in response to hypercapnia in 5 and 16 month-old mdx and C57 mice J Muscle Res Cell Motil. 2007;28(1):29-37.

94. Matecki S, et al. The effect of respiratory muscle training with CO2 breathing on cellular adaptation of mdx mouse diaphragm. Neuromuscul Disord. 2005;15(6):427-436.

95. Li SH, et al. Huntington's disease gene (IT15) is widely expressed in human and rat tissues. Neuron. 1993;11(5):985-993.

96. Hoogeveen AT, et al. Characterization and localization of the Huntington disease gene product. Hum Mol Genet. 1993;2(12):2069-2073.

97. Menuet C, et al. Catecholaminergic C3 neurons are sympathoexcitatory and involved in glucose homeostasis. $J$ Neurosci. 2014;34(45):15110-15122.

98. Menuet C, et al. Excessive respiratory modulation of blood pressure triggers hypertension. Cell Metab. 2017;25(3):739-748.

99. Tang H, Lee M, Khuong A, Wright E, Shrager JB. Diaphragm muscle atrophy in the mouse after long-term mechanical ventilation. Muscle Nerve. 2013;48(2):272-278

100. Mrozek S, et al. Rapid onset of specific diaphragm weakness in a healthy murine model of ventilator-induced diaphragmatic dysfunction. Anesthesiology. 2012;117(3):560-567.

101. Bevins RA, Besheer J. Object recognition in rats and mice: a one-trial non-matching-to-sample learning task to study 'recognition memory'. Nat Protoc. 2006;1(3):1306-1311

102. Schenk F, Morris RG. Dissociation between components of spatial memory in rats after recovery from the effects of retrohippocampal lesions. Exp Brain Res. 1985;58(1):11-28.

103. Amancio GCS, et al. Effect of pyridostigmine on in vivo and in vitro respiratory muscle of mdx mice. Respir Physiol Neurobiol. 2017;243:107-114.

104. Yin Z, et al. Low-fat diet with caloric restriction reduces white matter microglia activation during aging. Front Mol Neurosci. 2018;11:65

105. Cacheux M, et al. Interplay between triadin and calsequestrin in the pathogenesis of CPVT in the mouse. Mol Ther 2020;28(1):171-179.

106. Bouilloux F, et al. Loss of the transcription factor Meis1 prevents sympathetic neurons target-field innervation and increases susceptibility to sudden cardiac death. Elife. 2016;5:e11627.

107. Cutler TS, et al. Neurocardiovascular deficits in the Q175 mouse model of Huntington's disease. Physiol Rep. 2017;5(11):e13289. 